\title{
Characterization and generation of human definitive multipotent hematopoietic stem/ progenitor cells
}

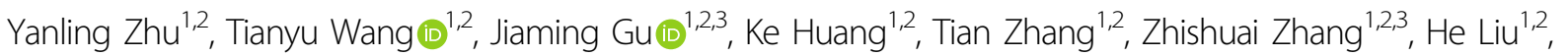 \\ Jun Tang ${ }^{1,2}$, Yuchan Mai ${ }^{1,2}$, Yanqi Zhang ${ }^{1,2,3}$, Yuhang Li, ${ }^{1,2,3}$, Yashu Feng ${ }^{4}$, Baogiang Kang ${ }^{1,2}$, Jinbing Li, $i^{1,2,3}$, \\ Yongli Shan 1,2, Qianyu Chen 1,2, Jian Zhang ${ }^{1,2}$, Bing Long ${ }^{4}$, Junwei Wang ${ }^{1,2}$, Minghui Gao ${ }^{1,2}$, Di Zhang ${ }^{1,2,3}$, Min Zhou ${ }^{1,2}$, \\ Xiaofen Zhong ${ }^{1,2}$, Jiekai Chen ${ }^{1,2}$, Duanqing Pei $\mathbb{D}^{1,2}$, Jinfu Nie ${ }^{1,2}$, Bing Liu $\mathbb{1}^{5}$ and Guangjin Pan $\mathbb{D}^{1,2,6,7}$
}

\begin{abstract}
Definitive hematopoiesis generates hematopoietic stem/progenitor cells (HSPCs) that give rise to all mature blood and immune cells, but remains poorly defined in human. Here, we resolve human hematopoietic populations at the earliest hematopoiesis stage by single-cell RNA-seq. We characterize the distinct molecular profiling between early primitive and definitive hematopoiesis in both human embryonic stem cell (hESC) differentiation and early embryonic development. We identify CD44 to specifically discriminate definitive hematopoiesis and generate definitive HSPCs from hESCs. The multipotency of hESCs-derived HSPCs for various blood and immune cells is validated by single-cell clonal assay. Strikingly, these hESCs-derived HSPCs give rise to blood and lymphoid lineages in vivo. Lastly, we characterize gene-expression dynamics in definitive and primitive hematopoiesis and reveal an unreported role of ROCK-inhibition in enhancing human definitive hematopoiesis. Our study provides a prospect for understanding human early hematopoiesis and a firm basis for generating blood and immune cells for clinical purposes.
\end{abstract}

\section{Introduction}

In embryogenesis, definitive hematopoiesis generates hematopoietic stem cells and multipotent progenitors (HSC/MPPs) that further give rise to all mature blood and immune cells. Classically, the multi-potent HSPCs undergo a stepwise differentiation to the oligo-, bi- and uni-potent progenitors of different types of mature blood cells, such as granulocyte-monocyte progenitors (GMPs), megakaryocyte-erythroid progenitors (MEPs), and

\footnotetext{
Correspondence: Guangjin Pan (pan_guangjin@gibh.ac.cn)

${ }^{1}$ CAS Key Laboratory of Regenerative Biology, Joint School of Life Sciences, Guangzhou Institutes of Biomedicine and Health, Chinese Academy of Sciences, Guangzhou Medical University, ,Guangzhou Guangdong 510530, China

${ }^{2}$ Guangdong Provincial Key Laboratory of Stem Cell and Regenerative Medicine, South China Institute for Stem Cell Biology and Regenerative Medicine, Guangzhou Institutes of Biomedicine and Health, Chinese Academy of Sciences, ,Guangzhou Guangdong 510530, China

Full list of author information is available at the end of the article

These authors contributed equally: Yanling Zhu, Tianyu Wang, Jiaming Gu
}

lymphoid progenitors $(\mathrm{LPs})^{1-7}$. However, many studies show that the uni-potent progenitors derive directly from HSPCs without going through oligo-potent progenitors, and thus the classical model of human hematopoiesis is revised $^{1,8-11}$. Nonetheless, the multipotent HSC/MPPs that conceptually initiate hematopoiesis are very rare in adult human blood system ${ }^{9,12,13}$. Human HSCs and MPPs are phenotypically known to locate within the $\mathrm{Lin}^{-} \mathrm{CD} 34^{+} \mathrm{CD} 38^{-}$compartment and both belong to a continuum of undifferentiated hematopoietic stem/progenitor cells (HSPCs) $)^{6,8,9,12,13}$.

During embryonic development, the multipotent HSPCs are originated in early definitive hematopoiesis through endothelial-to-hematopoietic transition (EHT) ${ }^{14-18}$. In the mouse model, the first wave hematopoiesis starts in yolk sac to generate primitive progenitors (mainly myeloerythroid) and then in aorta-gonad-mesonephros (AGM) region to generate definitive $\mathrm{HSPCs}^{14-19}$. These two 
waves of hematopoiesis generate functionally distinct progenitors, and the immune cells such as NK, lymphoid cells are mainly generated from the second wave of definitive hematopoiesis. To this end, human primitive and definitive hematopoiesis have not been well-characterized in early embryonic development. Human pluripotent stem cells (PSCs) such as embryonic stem cells (ESCs) and induced pluripotent stem cells (iPSCs) are useful models for investigation of human hematopoietic development in vitro $^{20-23}$. In hPSC differentiation, hemogenic endothelial cells (HECs) express CD34 and CD31 and then show upregulated CD43 on the commitment of hematopoietic fate ${ }^{22,24-26}$. CD43 has served as a marker to indicate hPSC-derived hematopoietic progenitors in many studies $^{23,24,27,28}$. However, even though these $\mathrm{CD} 43^{+}$ hPSC-derived HPCs could form various blood CFUs (colony-forming units), they have very limited definitive potency to give rise to immune cells ${ }^{23,25}$. Characterization of human primitive and definitive hematopoiesis at the earliest stage remains unaddressed, thus largely impeding the translational progress of human stem cell differentiation.

Here in this study, we resolve human early hematopoietic populations by single-cell RNA-seq and reveal distinct transcriptional profiling in early definitive and primitive hematopoiesis both in human embryonic stem cell (hESC) differentiation and early embryonic development. We identify CD44 to specifically discriminate the early definitive and primitive hematopoiesis. In addition, we generate definitive HSPCs from hESCs that give rise to various blood and immune lineages, including myeloid, erythroid/megakaryocyte cells, NK, T lymphocytes etc. and strikingly differentiate to various blood and lymphoid cells in vivo. Our studies provide a valuable model to understand human early hematopoietic development and generate various blood and immune cells for clinical purposes.

\section{Results}

\section{Single-cell transcriptional profiling of hematopoietic populations in hESC differentiation}

Based on reports from multiple groups ${ }^{20,29,30}$, we previously developed a stepwise strategy for hPSC blood differentiation in a totally defined, monolayer condition, which allows a robust EHT to generate $\mathrm{CD} 43^{+}$hematopoietic progenitors (hESCs-HPCs) that could form various blood $\mathrm{CFUs}^{28}$. However, the multi-potency of $\mathrm{CD} 43^{+}$ hESCs-HPCs in hematopoiesis both in vitro and in vivo remains questionable. To address this issue, we generated single-cell transcriptional profiling on FACS-sorted $\mathrm{CD}_{4} 3^{+}$hESCs-HPCs differentiated from H1 hESCs at day 8 based on the previous protocol ${ }^{28}$ (Fig. 1a). As a comparison, we also sequenced single cells of FACSsorted $\mathrm{Lin}^{-} \mathrm{CD} 34^{+} \mathrm{CD} 38^{-}$HSPCs mobilized in human peripheral blood (PB-HSPCs) (Fig. 1a). $t$-SNE visualization on single-cell transcription profiling displayed distinct clusters between PB-HSPCs and hESCs-HPCs (Fig. 1b, c). Consistent with previous findings ${ }^{9,13}$, most $\mathrm{Lin}^{-} \mathrm{CD} 34^{+} \mathrm{CD}^{-} 8^{-}$PB-HSPCs formed continuous rather than discrete pattern in transcriptional profiling (Fig. 1b, c). A small number of PB-HSPCs formed a separate cluster and expressed early lymphoid lineage markers such as CD10 and $\mathrm{LTB}^{5}$ (Fig. 1c, d), indicating they are lymphoid-primed HSPCs localized in $\mathrm{Lin}^{-} \mathrm{CD} 34^{+} \mathrm{CD} 38^{-}$compartment (PB-LPCs) ${ }^{6}$. The FACSsorted $\mathrm{CD} 43^{+} \mathrm{hESCs}$-HPCs formed an indiscrete cluster separated from both PB-HSPCs and PB-LPCs (Fig. 1b, c). Consistent with the FACS sorting, hESCs-HPCs displayed a uniform CD43 expression and were negative for most known lineage markers of different mature blood cells (Fig. 1d). Surprisingly, most of these CD $43^{+}$hESCs-HPCs highly expressed GATA1, a known transcription factor for early erythroid lineage $(\mathrm{Er})^{31}$, and NFE2, a myeloid (My) marker gene ${ }^{32}$ (Fig. 1d), indicating they might be Er/Mybiased progenitors rather than the multipotent ones. Indeed, genes for myeloid development such as MYL4, $D M T N$, and MPIG6B and platelet and erythroid development genes like ITGA2B, VCL, GATA1, HBG, and $H B D$ were highly enriched in hESC-HPC cluster (Fig. 1e, g). In contrast, known genes for definitive HSCs and/or lymphoid were highly enriched in PB-HSPC and PB-LPC clusters, respectively, such as ETV6, CD34, HLADR, LTB, HOXA9, and CCR7 (Fig. 1e, g). The top gene ontology (GO) terms enriched in hESC-HPC cluster were related to mitochondrial regulation, reflecting this cluster is related to erythroid lineage (Fig. 1g). Consistently, the function of lymphoid regulation was significantly enriched in PBLPCs (Fig. 1g). Together, we reveal that the marker-pure $\mathrm{CD} 43^{+}$hESCs-derived HPCs are mainly Er/My-biased progenitors rather than definitive ones based on geneexpression profiling, which has not been recognized before.

\section{hESCs-derived HPCs contain cells with different hematopoietic states}

To further characterize the $\mathrm{CD}_{4}{ }^{+}$hESCs-derived HPCs in detail, we applied pseudotime analysis to order all the hESC-HPCs based on their transcriptional profiling (Fig. 2a). Known genes that encode markers or regulators for human HSPCs such as CD34, CD90, MYB, RUNX1, GFI1, IKZF1, HOXA9, and GATA2 were downregulated along the pseudotime, whereas genes that encode the $\mathrm{Er} /$ My regulators such as GATA1, DMTN, VCL, and $H B A$ were significantly upregulated (Fig. 2b). Strikingly, genes with the function of lymphoid regulation such as $L T B$, LSP1, CCR7, were also downregulated along the pseudotime (Fig. 2b), indicating loss of definitive potential along pseudotime. We then divided hESC-HPCs into different 
a

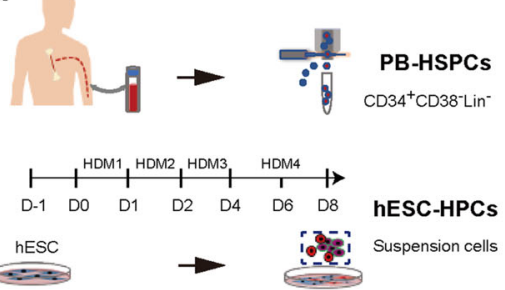

b

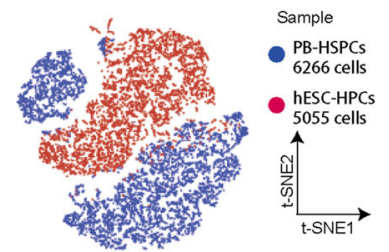

C

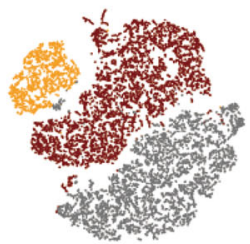

Cluster

-hESC-HPCS

PB-HSPCS

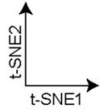

d
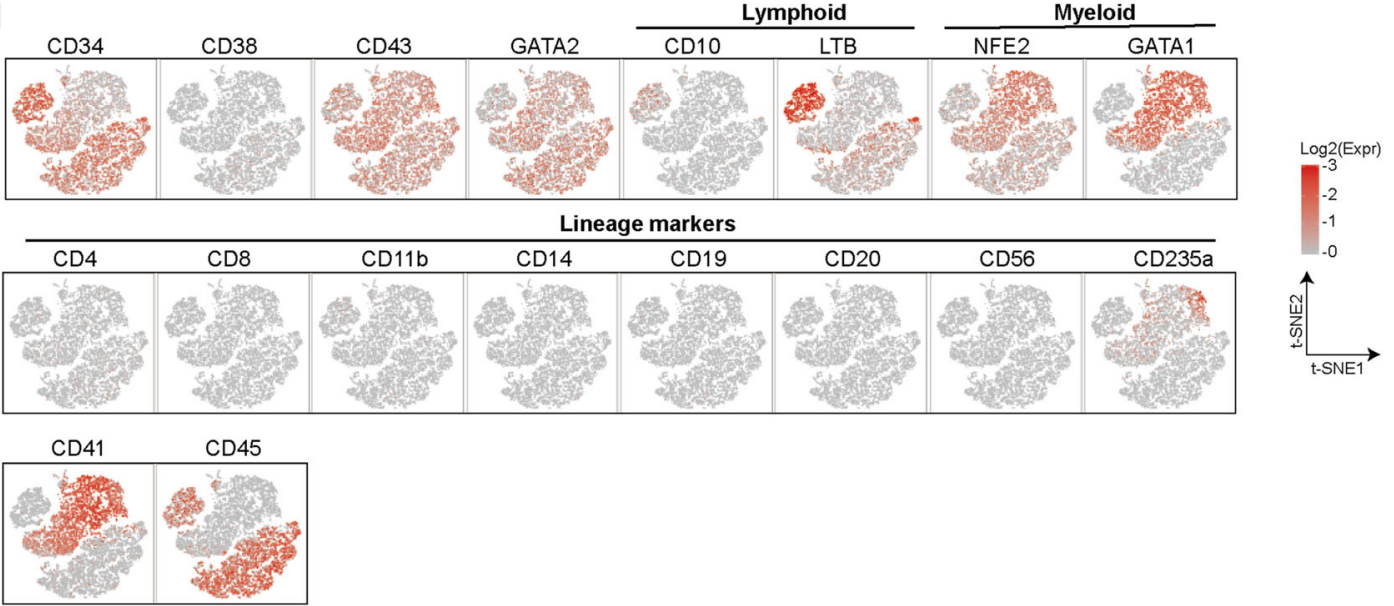

PB-LPCs

PB-LPCs

$\circ \circ \circ$

$\circ \circ$

$\circ \circ \circ$

hESC-HPCs

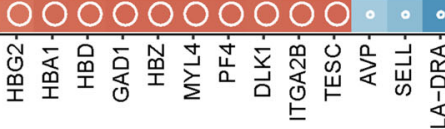

0000000000

0000000000

Fraction

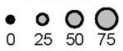

Expression

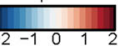

f

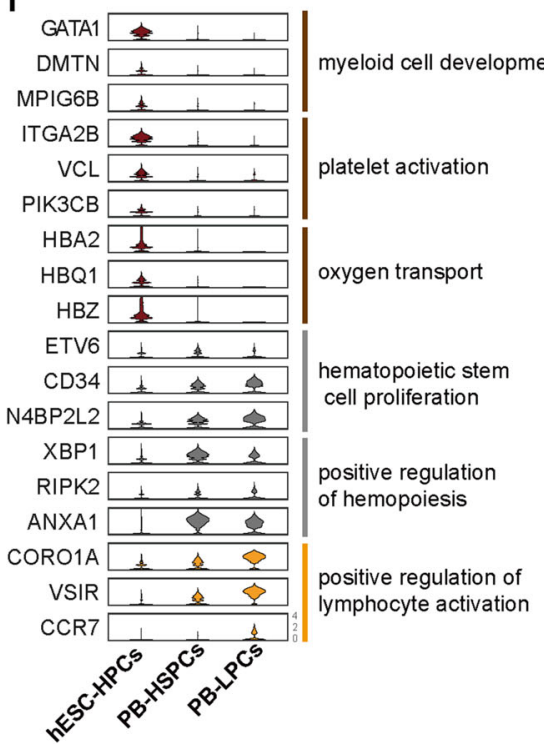

hESC-HPCs

mitochondrial inner membrane mitochondrial protein complex mitochondrial matrix inner mitochondrial membrane protein complex mitochondrial translational elongation

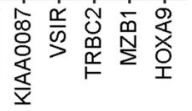

PB-LPCs
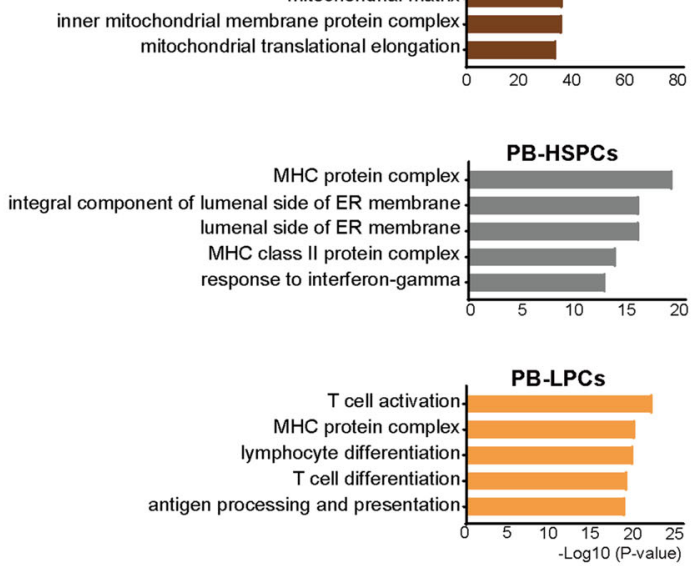

Fig. 1 (See legend on next page.) 
(see figure on previous page)

Fig. 1 Single-cell RNA sequencing of hPSCs-derived HPCs and adult peripheral blood HSPCs. a Scheme of the experiment design. Fresh $\mathrm{Lin}^{-} \mathrm{CD} 34^{+} \mathrm{CD}_{3}{ }^{-}$HSPCS mobilized in human peripheral blood (PB-HSPCs) were sorted by FACS. Human ESCs were differentiated into blood lineages in a monolayer, defined condition. The floating blood cells at differentiation day 8 were sorted by CD43. The sorted cells were analyzed by 10x genomics for single-cell RNA sequencing (scRNA-seq). $\mathbf{b} t$-SNE projection of PB-HSPC and hESC-HPC sample cells assigned based on samples. Each dot represents one cell and colors represent cell samples. $\mathbf{c}$-SNE projection of hESC-HPC, PB-HSPC, and PB-LPC cluster cells assigned based on clusters. Each dot represents one cell and cells are colored according to their assigned clusters. $\mathbf{d}$ Expressions of known hematopoietic and blood lineage marker genes at single-cell resolution. Color displays expression level (TPM, Log-scaled). e Expression profiles of top highly expressed genes in hESC-HPC, PB-HSPC and PB-LPC clusters. Color displays the scaled expression level (TPM, z-normalized) and diameter denotes fractional expression. f Violin plots show expression levels (TPM, Log-scaled) of selected hematopoietic and blood lineage marker genes with GO terms enriched in hESCHPC, PB-HSPC and PB-LPC clusters. Colors represent clusters. g Top Gene Ontology (GO) terms enriched in hESC-HPC, PB-HSPC, and PB-LPC clusters. Colors represent clusters. The length of bar represents $P$ value.

clusters based on pseudotime progression (Fig. 2c). Consistently, genes that encode markers or regulators of HSCs such as CD34, CD90, MYB, RUNX1, GFI1, IKZF1, HOXA9, and GATA2 were highly expressed in the early stage of cells but significantly downregulated in the cells at the middle and late stage in pseudotime progression (Fig. 2d). In contrast, the Er/My genes were expressed at lower levels in early stage cells, but their expression significantly increased in middle and late-stage cells (Fig. 2d). Again, lymphoid genes were highly expressed in the cells at an early stage but their high expressions were lost at later stages in pseudotime progression (Fig. 2d). These data indicate that cells at the early stage of pseudotime progression are less-primed definitive hematopoietic progenitors. Indeed, pearson co-efficiency analysis clearly showed a much closer relationship between the early stage hESC-HPCs and PB-HSPCs, compared with the cells at the middle and late stages (Fig. 2e). Top dynamic genes that were downregulated at later stage cells include those encode many known HSPCs or stem cell regulators such as ID1, CD34, GATA2, HOPX, and also those encode other new factors (Fig. 2f-h). Notably, GATA1, the known Er regulator gene displayed a mutually exclusive pattern with $L T B$, the lymphoid gene, and most of the other HSPC-related genes along pseudotime progression (Fig. $2 \mathrm{~g}-\mathrm{h}$ ), again demonstrating the FACS-sorted CD43 ${ }^{+}$ marker-pure hESCs-HPCs are heterogeneous and contain cells with different hematopoietic states.

\section{Identification of definitive multi-potent HSPCs in hESC differentiation}

Since the cell surface marker CD44 and GATA1 are almost mutually exclusive in their expression and display opposite patterns during pseudotime progression (Fig. 2g), we reasoned that CD44 might specifically label the low-primed definitive HSPCs. Based on a digital cytometry (d-cyto) analysis using single-cell transcriptional profiling, $85 \%$ of PB-HSPCs are shown to be CD $44^{+}$GATA1 $^{-}$(Fig. 3a, b). In contrast, only $6.2 \% \mathrm{CD} 43^{+}$ hESCs-HPCs were CD44 $4^{+}$GATA1 $^{-}$(Fig. 3a, b). These findings indicate that CD44 might be a previously un- recognized surface marker to label definitive multi-potent HSPCs in differentiation. We then performed flow cytometry analysis (FACS) to examine CD44 expression in a different batch of $\mathrm{Lin}^{-} \mathrm{CD} 34^{+} \mathrm{CD} 38^{-}$PB-HSPCs as well as hESCs-derived HPCs. Consistent with d-cyto data, near $100 \%$ of fresh $\mathrm{Lin}^{-} \mathrm{CD} 34^{+} \mathrm{CD} 38^{-} \mathrm{PB}-\mathrm{HSPCs}$ were CD44 positive, whereas only around $50 \%$ of $\mathrm{CD} 43^{+}$hESCsHPCs were CD44 positive (Fig. 3c). We then analyzed the multi-potency of $\mathrm{CD}_{4} 4^{+}$hESCs-HPCs in more detail through both in vitro and in vivo approaches (Fig. 3d). Firstly, based on methylcellulose (MC) colony-forming assays (MC-CFUs), the FACS-sorted $\mathrm{CD} 43^{+} \mathrm{CD} 44^{+}$ hESCs-HPCs showed significantly higher CFU efficiencies than the $\mathrm{CD} 43^{+} \mathrm{CD} 44^{-}$cells (Fig. $3 \mathrm{e}^{-}$g). Moreover, CFUs formed by $\mathrm{CD} 43^{+} \mathrm{CD} 44^{-}$cells were very small and mainly erythroid ones (Fig. 3e-g). In contrast, $\mathrm{CD} 43^{+} \mathrm{CD} 44^{+}$cells formed big CFUs with various subtypes including CFU-E, CFU-G (granulocytes), CFU-M (macrophages) and many CFU-Mix subtypes (Fig. $3 \mathrm{e}^{-\mathrm{g}}$ ). Since the standard MC$\mathrm{CFU}$ assays are less efficient for detecting myeloid and lymphoid potential at the same time, we then examined $\mathrm{CD} 43^{+} \mathrm{CD} 44^{+}$cells through co-culturing with MS-5 stromal cells, which support the development of both human myeloid and lymphoid in vitro ${ }^{6,13}$. Upon coculturing with MS-5 cells in the presence of cytokines, $\mathrm{CD} 43^{+} \mathrm{CD} 44^{+}$cells gave rise to significant amount of myeloid cells as detected by $\mathrm{CD}^{+} 5^{+}$(granulocytes) and $\mathrm{CD}^{+} 4^{+}$(macrophages) (Fig. 3h). Moreover, we could detect a significant megakaryocyte-erythroid (Mk-Er) population in $\mathrm{CD} 43^{+} \mathrm{CD} 44^{+}$cells co-culturing with MS5 (Fig. 3h),consistent with the recent report that the Mk-Er lineage could directly differentiate from human adult HSPCs $^{8}$. More importantly, we detected significant $\mathrm{CD} 6^{+} \mathrm{NK}$ cells in the differentiation (Fig. 3h), indicating $\mathrm{CD} 43^{+} \mathrm{CD} 44^{+}$cells hold lymphoid potential. Furthermore, through OP9-DL4 stromal cell co-culturing that supports $\mathrm{T}$ cell differentiation, we detected $\mathrm{CD} 4$ and/or $\mathrm{CD} 8$ positive $\mathrm{T}$ cells derived from the $\mathrm{CD} 43^{+} \mathrm{CD} 44^{+}$cells (Fig. 3h). Together, these data demonstrate that $\mathrm{CD} 43^{+} \mathrm{CD} 44^{+}$labels definitive multipotent HSPCs in hESC differentiation (hESCs-HSPCs). 


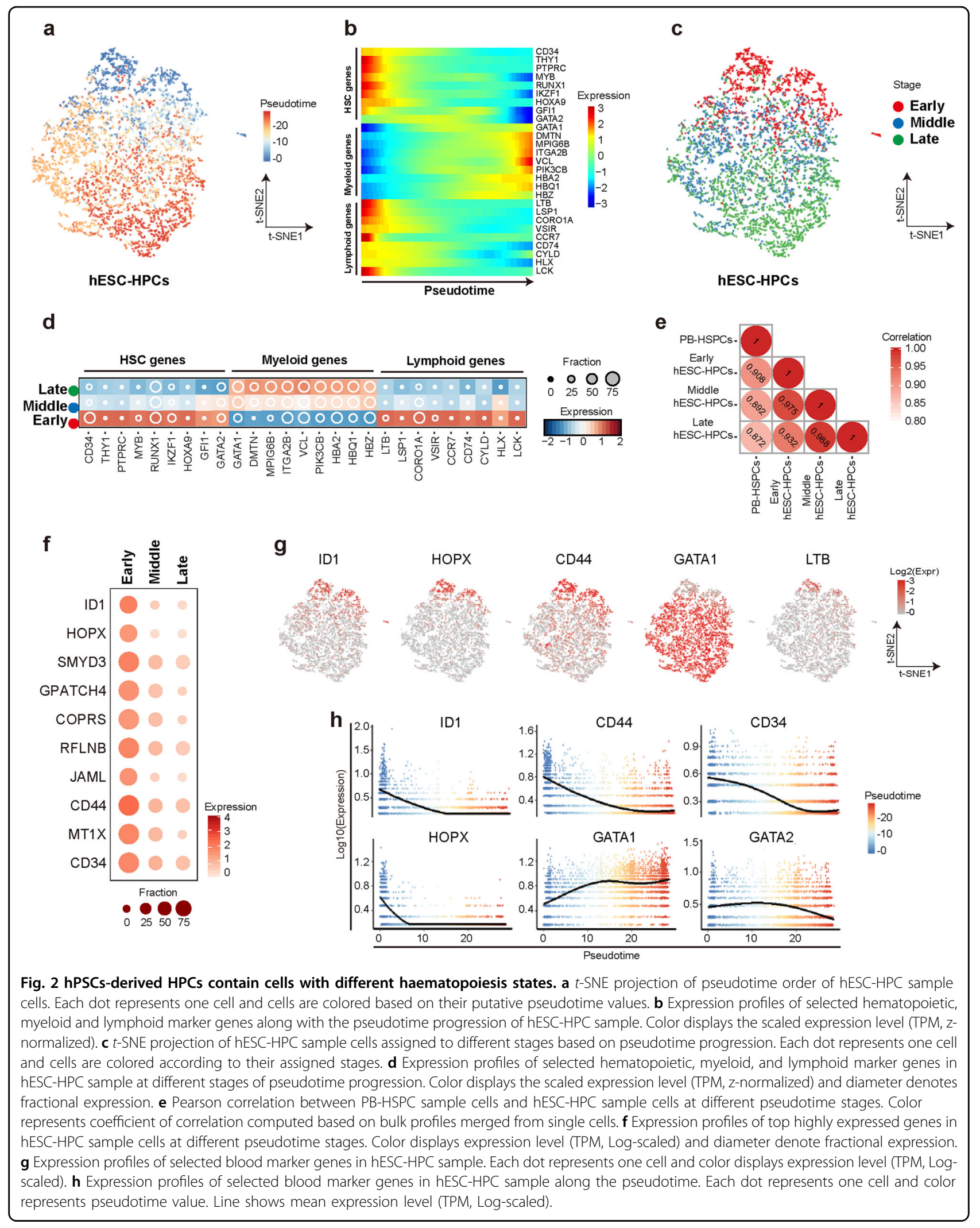




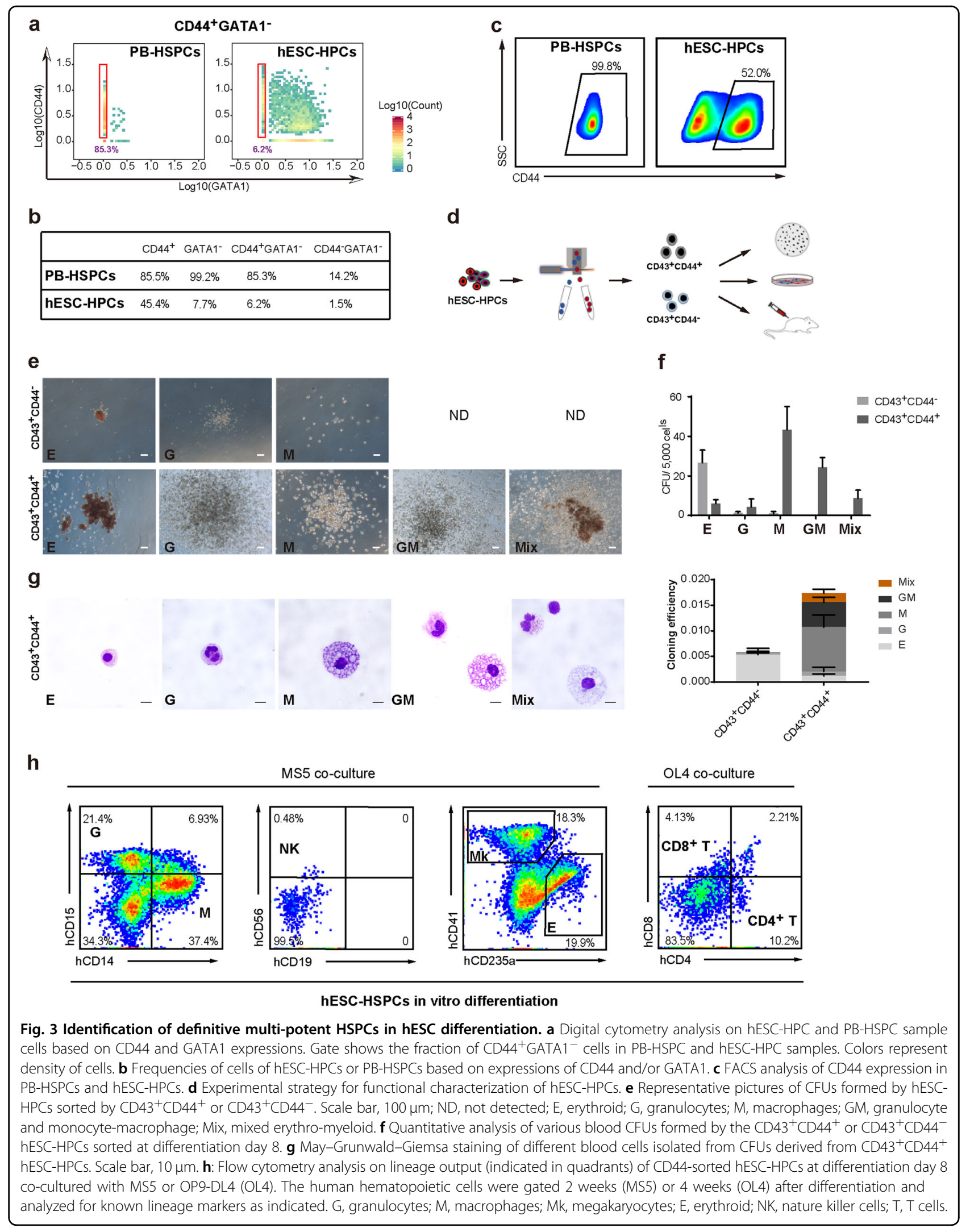


Clonal assays of lineage potential of definitive hESC-HSPCs

Examining lineage fate potential at the single-cell level is a golden standard to assess the multi-potency of HSPCs. We then investigated the lineage potential of $\mathrm{CD} 44^{+}$ hESC-HSPCs at single-cell level through MS-5 co-culturing ${ }^{6,13}$ (Fig. 4a, b). The clonal outputs of major lineages including myeloid (My), lymphoid (Ly/NK), erythroidmegakaryocyte (Er-Mk) were analyzed based on known surface markers (Fig. 4c, d). Single CD $43^{+} \mathrm{CD} 44^{+}$hESCsHSPCs seeded in this condition yielded uni- and multipotential blood colonies with the overall output clonal efficiency around 15\% (Fig. 4c, d). Specifically, the percentage of uni-lineage potential for each individual blood lineage (Er only, My only, Mk only and Ly/NK only) was quite even, which was around $10 \%$ of total blood colonies for each (Fig. 4c, d). Strikingly, more than half of single cell-derived blood colonies were bi- and tri-lineage potential (Fig. 4c, d). Notably, the total output potential for each individual lineage such as Er, My, Mk and Ly/NK from all the uni-, bi- and tri-lineage colonies varied a lot between different lineages (Fig. 4e). More than $60 \%$ of total single hESC-HSPC-derived blood colonies contained either Er and/or Mk, whereas around 15\% contained Ly/ NK lineage (Fig. 4e), indicating an Er/Mk predominance. These features are quite consistent with those of human HSPCs in the early fetal liver that were recently reported to exhibit differential intrinsic lineage potential according to the gestational stage, i.e., with early Er predominance and later Ly representation ${ }^{33}$. Nonetheless, we demonstrate that CD44-labeled hESC-derived definitive HSPCs hold the multi-potency to give rise to various blood cells and immune cells based on single-cell clonal assays. Furthermore, limiting-dilution assays (LDA) generated a similar estimate on the lineage potential of myeloid and/ or lymphoid in hESCs-HSPCs (Fig. 4f).

\section{Definitive hESC-HSPCs give rise to various blood and lymphoid lineages in vivo}

To further examine the lineage potential in vivo, we transplanted FACS-sorted CD $43^{+} \mathrm{CD} 44^{+}$hESCs-HSPCs directly into the tibia of irradiated NSI mice (Fig. 4g). We detected a significant number of $\mathrm{CD} 45^{+}$human cells from the bone marrow (BM) of the injected tibia 2 weeks after transplantation (Fig. 4h). The engrafted CD45 $5^{+}$human cells varied from near 1\% to $3 \%$ of total cells isolated from mouse tibia and contained large proportion of $\mathrm{CD} 15^{+}$ granulocytes, $\mathrm{CD} 14^{+}$macrophages as well as lymphoid lineages such as $\mathrm{CD} 19^{+} \mathrm{B}$ cells and $\mathrm{CD} 56^{+}$NK cells (Fig. $4 \mathrm{~h}-\mathrm{j}$ ). In addition, a large number of Mk-Er lineage progenitors could also be detected in human engraftment (Fig. 4j). Interestingly, we could detect a significant number of human B and NK lineages in BM of noninjected tibia (Fig. 4h, i), indicating homing and migration of the transplanted hESC-HSPCs in vivo. Together, these data demonstrate that the $\mathrm{CD} 43^{+} \mathrm{CD} 44^{+}$hESCs-HSPCs could give rise to multiple lineages in vivo upon transplantation into mice.

\section{Discrimination of earliest primitive and definitive hematopoiesis in hESC differentiation}

As reported by other groups, both the primitive and definitive hematopoiesis occur in hPSC differentiation ${ }^{20,21}$, but remain poorly defined due to the limited reliable markers. To track the origin of the definitive CD44 hESCs-HSPCs, we analyzed the EHT in our monolayer hematopoietic differentiation. To identify cells that were undergoing EHT, we co-stained the differentiated cells at day 8 for demonstrating the endothelia cell marker, CD31, and the hematopoietic cell markers, CD43 as well as CD44. Consistent with previous reports, most round but attached cells were co-stained for both CD31 and CD43 (Fig. 5a), showing the typical phenotype of cells undergoing EHT. However, a significant number of the nascent CD43 ${ }^{+}$cells undergoing EHT were negative for CD44 (Fig. 5a). The EHT cells were then analyzed by FACS (Fig. 5b). The CD31-gated cells were clearly separated into 4 distinct groups based on CD44 and/or CD43 staining: $\mathrm{CD} 31^{+} \mathrm{CD} 43^{-} \mathrm{CD} 44^{-}, \quad \mathrm{CD} 31^{+} \mathrm{CD} 43^{-} \mathrm{CD} 44^{+}, \quad \mathrm{CD} 31^{+}$ $\mathrm{CD} 43^{+} \mathrm{CD} 44^{+}$and $\mathrm{CD} 31^{+} \mathrm{CD} 43^{+} \mathrm{CD} 44^{-}$. (Fig. 5b). Two cell groups with EHT phenotype, $\mathrm{CD} 31^{+} \mathrm{CD} 43^{+} \mathrm{CD} 44^{+}$ and $\mathrm{CD} 31^{+} \mathrm{CD} 43^{+} \mathrm{CD} 44^{-}$showed distinct expressions of hematopoiesis related genes (Fig. 5c). Strikingly, the primitive hematopoiesis genes such as GATA1, HBA2 and $H B E$ were highly expressed in $\mathrm{CD} 31^{+} \mathrm{CD} 43^{+} \mathrm{CD} 44^{-}$cells, whereas they were not expressed or expressed at low levels in $\mathrm{CD} 31^{+} \mathrm{CD} 43^{+} \mathrm{CD} 44^{+}$cells (Fig. 5c). In contrast, the expressions of HSC-related genes like RUNX1, MYB, and SPI1 were much higher in $\mathrm{CD} 31^{+} \mathrm{CD} 43^{+} \mathrm{CD} 44^{+}$cells than those in $\mathrm{CD} 31^{+} \mathrm{CD} 43^{+} \mathrm{CD} 44^{-}$cells (Fig. 5c). These data demonstrate that $\mathrm{CD} 44$ efficiently discriminates definitive hematopoiesis from the primitive ones at the earliest stage of human hematopoiesis. As expected, the $\mathrm{CD} 43^{-}$cells, either $\mathrm{CD} 44^{+}$or $\mathrm{CD} 44^{-}$, expressed high endothelia genes such as CD144, CLDN5, and CAV1 (Fig. 5c), consistent with their endothelia phenotype. Furthermore, the sorted $\mathrm{CD}_{4} 4^{+}$definitive HSPCs showed much better expansion than the $\mathrm{CD} 44^{-}$primitive progenitors in typical medium supporting human HSPCs (Fig. 5d). Functionally, CD $44^{+}$HSPCs could give rise to big CFU-E as well as many other lineages, whereas CD44- cells only produced small CFU-E and very little myeloid cells (Fig. 5e). Furthermore, the adult globin gene like $H B B$ was highly expressed in $C D 44^{+}$ HSPCs-derived erythroid cells but not in CD44cells-derived erythrocytes (Fig. 5f). In contrast, the embryonic globin genes such as $H B E$ and $H B G 1$, were highly expressed in $\mathrm{CD} 44^{-}$cells-derived erythroid cells, but their expressions were much lower in CD $44^{+}$ 
a

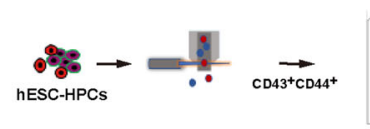

MS5 stromal cell b

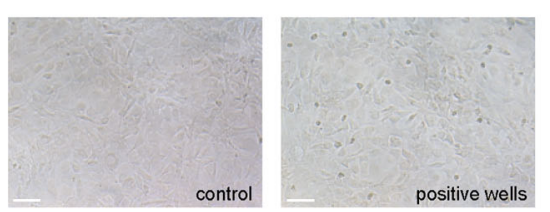

C
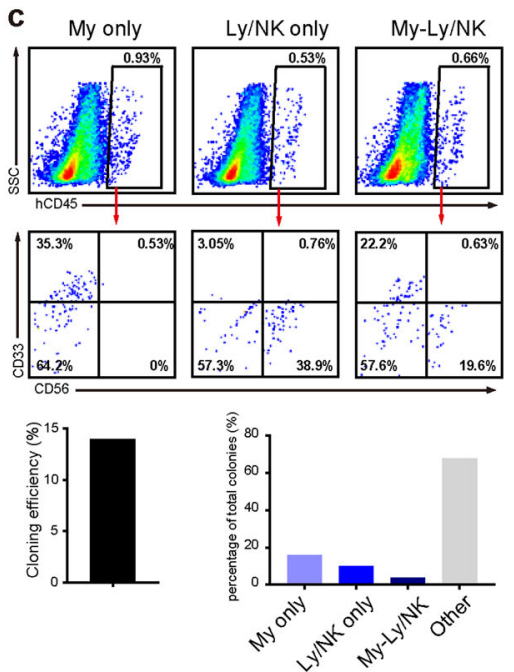

e

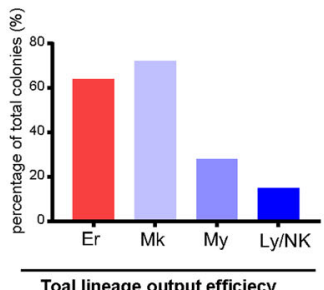

g
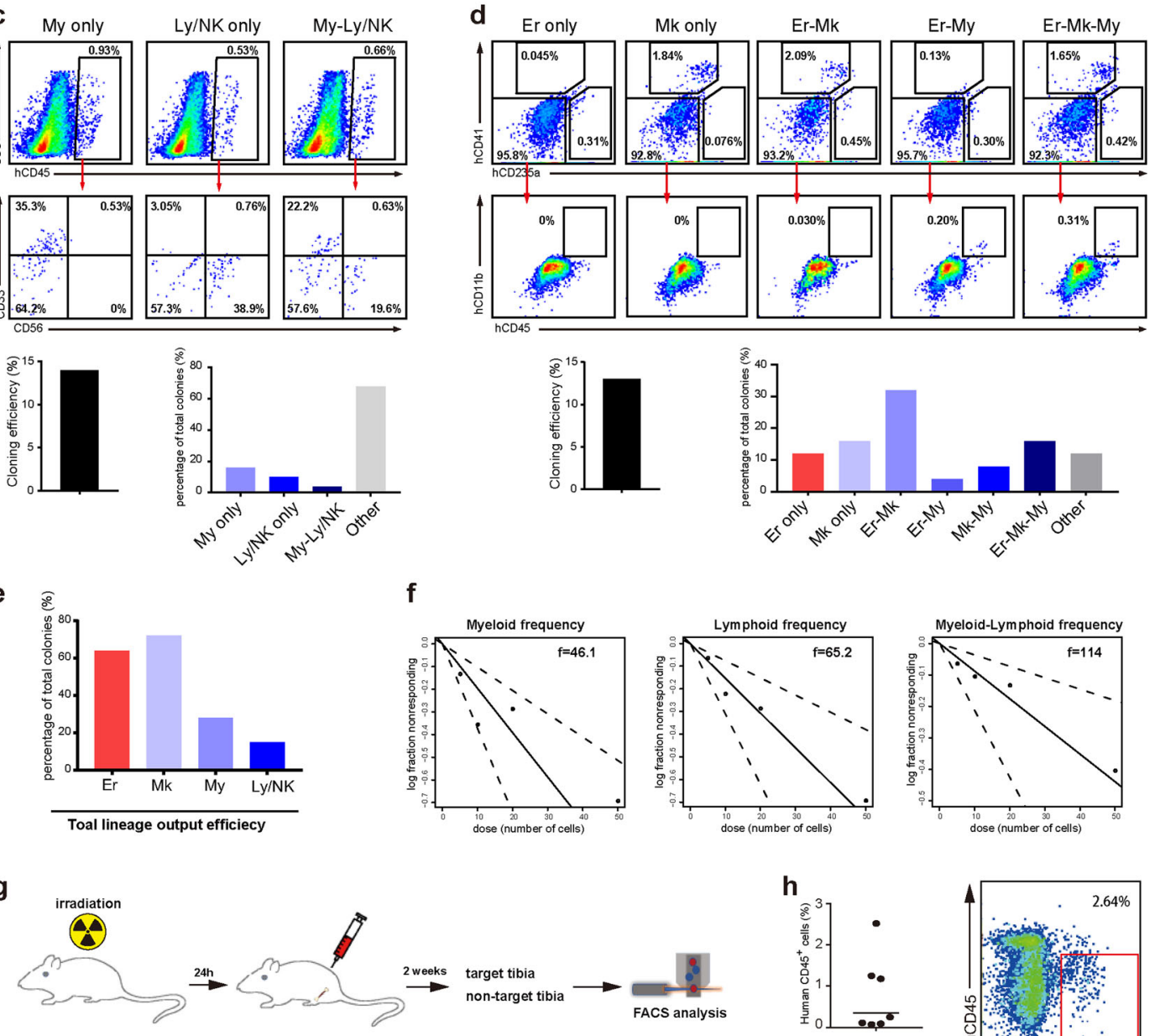

i

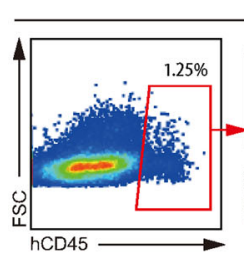

target tibia BM
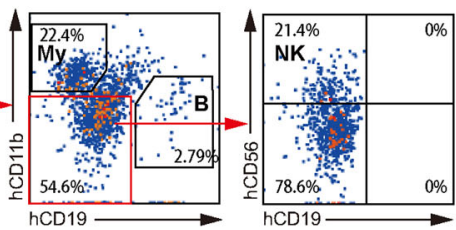

j
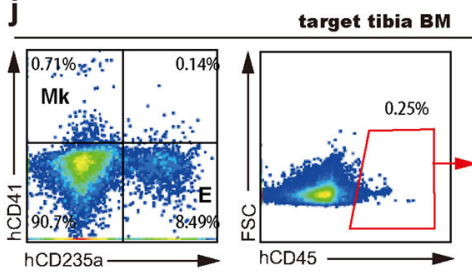

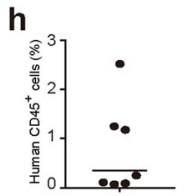

target tibia ( 2 weeks)

non-target tibia BM

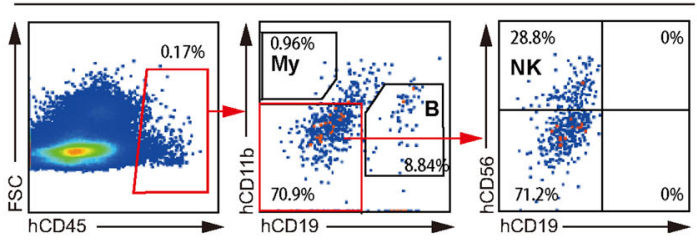

Fig. 4 (See legend on next page.) 


\begin{abstract}
(see figure on previous page)
Fig. 4 Analysis of multi-potency of hESC-HSPCs by single-cell clonal assay and in vivo transplantation. a Experimental design of clonal assays of lineage potential of hESC-HSPCs co-culturing with MS-5. CD43 ${ }^{+} \mathrm{CD}_{4} 4^{+}$hESC-HSPCs were sorted at differentiation day 8 and seeded on MS-5 cells in 10 96-wells plates at single-cell level. Myeloid and/or lymphoid lineage outputs were examined 4 weeks after co-culturing. b Morphology of single hESC-HSPCs co-culturing with MS-5 for 4 weeks. Scale bar: $100 \mu \mathrm{m}$. c FACS analysis of myeloid and/or lymphoid lineage outputs of single hESC-HSPCS co-culturing with MS-5. The CD45+ human hematopoietic cells were gated 4 weeks after differentiation and analyzed for known lineage markers as indicated. CD33 for myeloid and CD56 for lymphoid/NK. Left lower panel: quantitative data of total cloning efficiency of single hESC-HSPCs that can form blood clones. Right lower panel: percentage of myeloid and/or lymphoid clones of total formed blood clones by single hESC-HSPCS. $\mathbf{d}$ FACS analysis of erythroid, megakaryocyte and/or myeloid lineage outputs of single hESC-HSPCs co-culturing with MS-5. The cells were gated 4 weeks after differentiation and erythroid clones were defined as hCD235a ${ }^{+}$only (Er only). Megakaryocyte clones were defined as hCD41 ${ }^{+}$only (Mk only). Erythroid/megakaryocyte clones were defined as hCD235a ${ }^{+}$and hCD $41^{+}$but negative for hCD11b (Er/Mk). Erythroid/myeloid clones were defined as hCD235a $a^{+}$and hCD11 $b^{+}$but negative for hCD41 (Er/My). Erythroid/megakaryocyte/ myeloid clones were defined as hCD235a ${ }^{+}, h C D 41^{+}$and hCD $11 b^{+}$(Er/Mk/My). Left lower panel: quantitative data of total cloning efficiency of single hESC-HSPCs that can form blood clones. Right lower panel: percentage of erythroid, megakaryocyte, and/or myeloid clones of total formed blood clones by single hESC-HSPCs. e Lineage cloning efficiency of single hESC-HSPCs shown in $\mathbf{c}$ and $\mathbf{d}$, presented as the proportion of cells of various lineages in positive wells. Er, Erythroid potential (erythroid plus mixed); MK, Megakaryocyte potential (megakaryocyte plus mixed); My, Myeloid potential (myeloid plus mixed); Ly/NK, Lymphoid potential (lymphoid plus mixed). $\mathbf{f}$ Limiting-dilution assays for myeloid and/or lymphoid frequencies of hESC-HSPCs. LDA plots show the frequency (f: 1 in X cells can give rise to) of CD44 ${ }^{+}$hESC-HSPCs in myeloid, lymphoid and myeloid-lymphoid potential. Plots are generated by ELDA software. $\mathbf{g}$ Experimental design of in vivo transplantation of hESC-HSPCs. $\mathbf{h}$ Human CD45 $5^{+}$cell engraftment in the injected tibias of NSI mice 2 weeks after transplantation of hESC-HSPCs. $\mathbf{i}, \mathbf{j}$ In vivo lineage potential of hESC-HSPCs. CD43 ${ }^{+}$CD $44^{+}$hESC-HSPCs were sorted at differentiation day 8 and transplanted into the tibia of NSI mice. Multilineage outputs were examined 2 weeks after transplantation. Myeloids (My), Nature killer cells (NK), B cells, Granulocytes (G) and macrophages (M) were gated for human $\mathrm{CD}_{4} 5^{+}$events. Megakaryocytes and erythrocytes were defined as hCD41 ${ }^{+}$only (Mk) or hCD235a+ (E) only.
\end{abstract}

cells-derived ones (Fig. 5f). Together, all these data demonstrate that CD44 expression discriminates the earliest definitive and primitive hematopoiesis in hESC differentiation.

\section{Human definitive and primitive hematopoiesis in early embryonic development}

Definitive and primitive hematopoiesis have so far not been well characterized in human early embryonic development. To address this issue, we analyzed singlecell transcriptional profiling generated from early human embryonic samples at Carnegie stage 10-13(CS10-13), 23-30 days post coitus (dpc) ${ }^{34}$ (Fig. 6, Supplementary Fig. S1). Based on the expression of known endothelia and hematopoietic marker genes, such as SPI1, CD34, RUNX1, $C D H 5$, we extracted endothelia and hematopoietic cells from early human embryonic samples (Fig. 6a, Supplementary Fig. S1). We identified a cluster of cells coexpressing both endothelia and hematopoietic genes, indicating they are nascent hematopoietic cells in the human early embryo (Fig. 6b, c). These cells were further divided into three distinct clusters based on their transcriptional profiling (Fig. 6c). One cluster is obviously the primed erythroid fate as the cells highly expressed primitive erythroid genes such as GYPA, HBG1. (Fig. 6d). The other two clusters are nascent low-primed HSPCs as they express HSPC-related genes like CD34, GATA2, $M Y B, \quad R U N X 1$, and/or CDH5, the endothelia gene (Fig. 6d). Strikingly, CD44 and GATA1 showed clearly mutually exclusive expressions in two groups of lowprimed HSPCs in the human embryo (Fig. 6d). Genes highly expressed in $\mathrm{CD} 44^{+}$group cells were significantly enriched for functions related to HSC regulation and particularly the lymphoid potential (Fig. 6e), demonstrating these cells are nascent definitive HSPCs in human early development. In contrast, genes in CD44 group cells were related to Er/My fate, whereas they showed no lymphoid related function, suggesting this group of cells might be mainly primitive Er/My progenitors in human development. Indeed, selected $\mathrm{Er} / \mathrm{My}$ and HSPC genes showed distinct expression patterns in $\mathrm{CD} 44^{+}$definitive and CD $44^{-}$primitive cells (Fig. 6f). Particularly, lymphoid genes were significantly enriched in $\mathrm{CD} 44^{+}$definitive HSPCs compared with the CD44- primitive ones (Fig. 6f). Together, these data suggest that the earliest definitive and primitive hematopoiesis in human early embryonic development exhibit differential CD44 expression patterns.

\section{ROCK-inhibition promotes human definitive hematopoiesis}

The identification of CD44 to discriminate the definitive and the primitive hematopoiesis allows us to investigate pathways to regulate these two functionally distinct waves of hematopoiesis. Based on the selected marker genes, the pseudotime progression generated from all the $\mathrm{CD} 43^{+}$ hESCs-derived cells represent a fate change from a lowprimed definitive state to primitive erythroid fate (Fig. 2b). We, therefore, characterized dynamic genes along with the pseudotime progression (Fig. 7a, b). Consistently, genes with their expression increased along pseudotime are highly related to myelo-erythroid fate while those downregulated genes are related to lymphocyte fate regulation, cell cycle, etc. (Fig. 7c), reflecting a gradual 


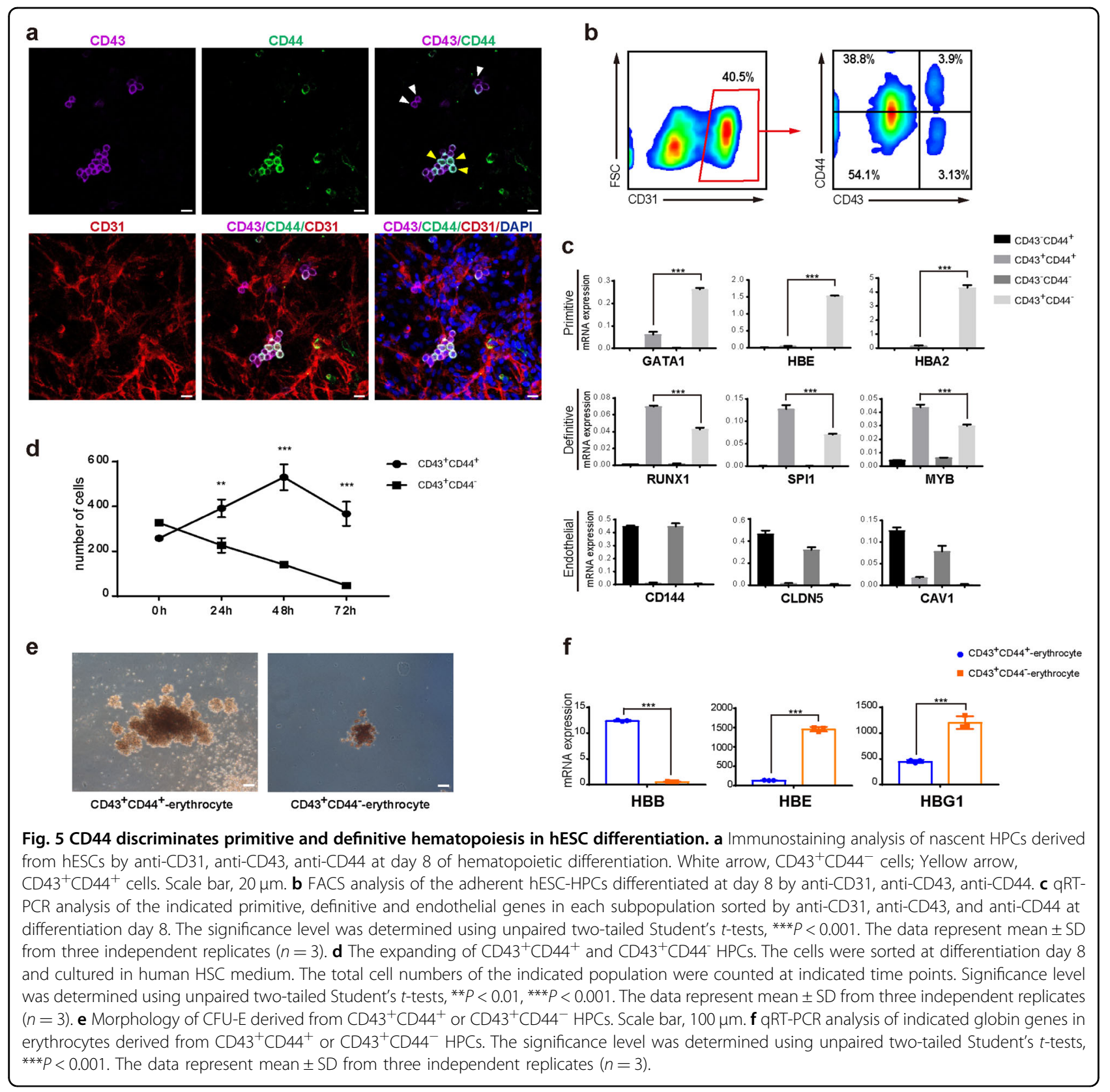

definitive to primitive fate change. We identified signaling pathways that were differentially regulated between human primitive and definitive hematopoiesis (Fig. 7d). Many of these pathways are known to play important roles in hematopoiesis, such as MAPK, VEGF ${ }^{35-38}$ (Fig. $7 \mathrm{~d})$. We noted that ROCK pathway genes showed an obvious upregulation in fate change from definitive to primitive hematopoiesis (Fig. 7d, e), but their roles in human hematopoiesis has not been recognized before. Interestingly, these ROCK signaling genes also exhibited differential expression in early definitive and primitive
HSPCs identified in the human embryo (Figs. 6, 7f). We further confirmed that the expressions of these ROCK pathway genes were significantly higher in $\mathrm{CD} 43^{+} \mathrm{CD} 44^{-}$ primitive progenitors than those in $\mathrm{CD} 43^{+} \mathrm{CD} 44^{+}$definitive ones in hESC differentiation (Fig. 7g). These data indicate that ROCK signaling positively regulates early primitive hematopoiesis in humans. To further examine the role of ROCK in human hematopoiesis, we added the known chemical inhibitor for ROCK (Y-27632) during EHT process in hESC differentiation (Fig. $7 \mathrm{~h}$ ). Indeed, the percentage of definitive progenitors that were either 
a

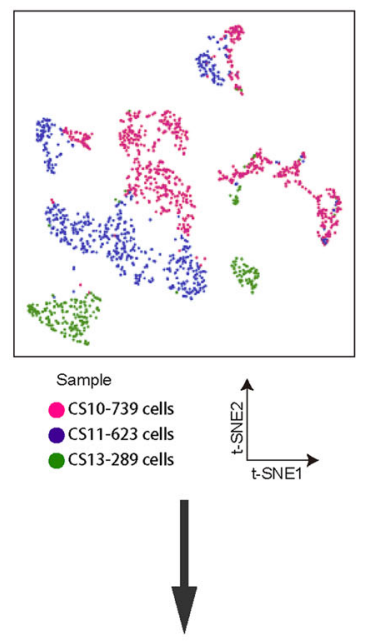

c
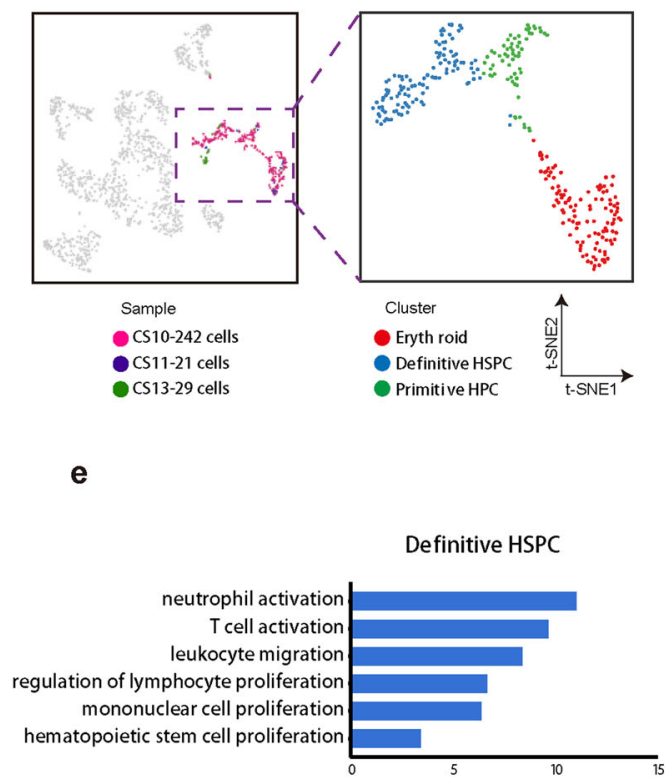

Primitive HPC

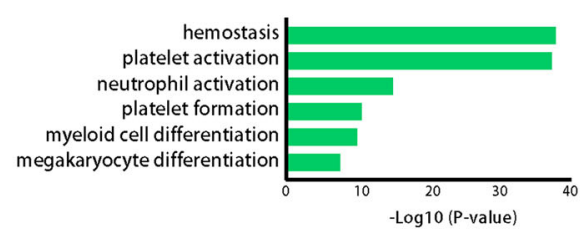

b Endothelial genes Hematopoietic genes

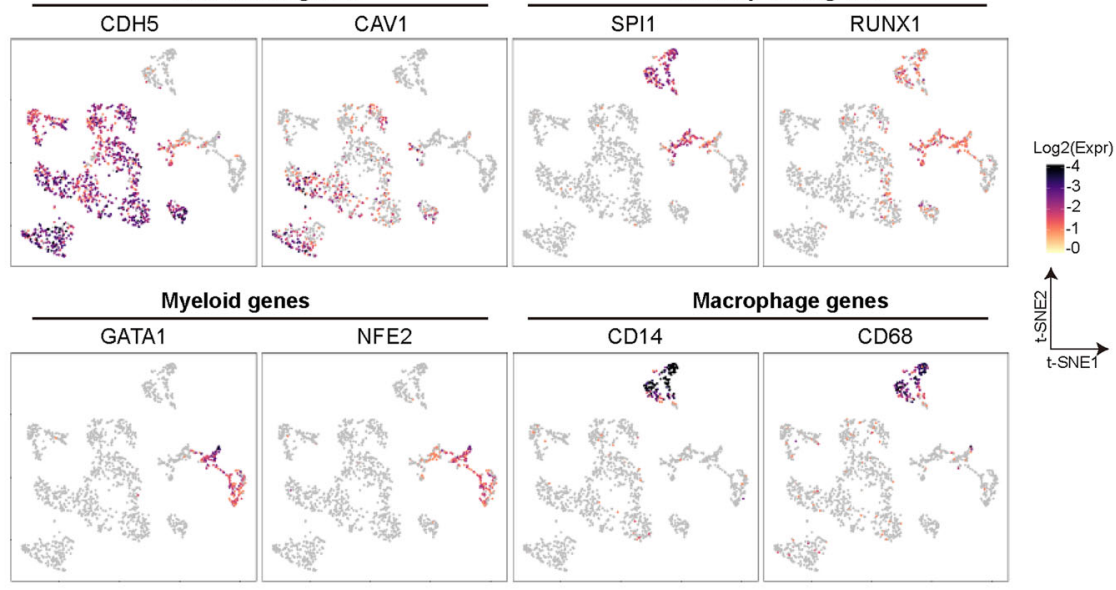

d

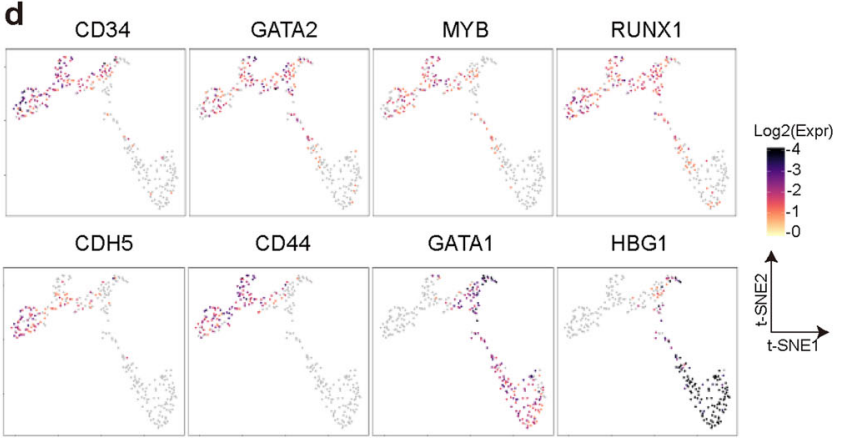

f
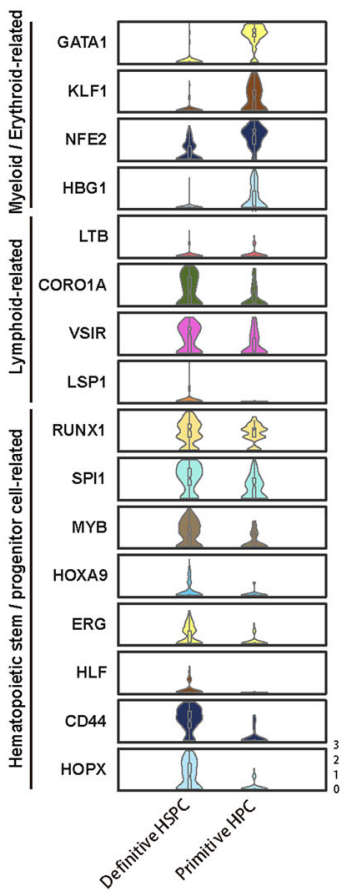

Fig. 6 (See legend on next page.) 
(see figure on previous page)

Fig. 6 Definitive human hematopoiesis in early embryonic development. a $t$-SNE projection of endothelial and hematopoietic cells, resulting from sub-dividing the cells in Supplementary Fig. S1a as indicated in Supplementary Fig. S1c, assigned based on samples. Each dot represents one cell and colors represent cell samples. The legend shows the number of cells in each sample. $\mathbf{b}$ Expressions of known blood and endothelial marker genes at single-cell resolution. Color displays expression level (TPM, Log-scaled). c $t$-SNE projection of erythroid, definitive HSPC and primitive HPC cluster cells, resulting from sub-dividing the cells in Fig. 6a as indicated in the left panel, according to blood and endothelial marker gene expression as indicated in Fig. 6b. d Expression profiles of selected feature genes in erythroid, definitive HSPC and primitive HPC cluster cells. Each dot represents one cell and color displays expression level (TPM, Log-scaled). e Top Gene Ontology (GO) terms enriched in definitive HSPC and primitive HPC clusters. Colors represent cell clusters. f Violin plots show expression levels (TPM, Log-scaled) of selected myeloid/erythroid, lymphoid and hematopoietic stem/progenitor cell marker genes in definitive HSPC and primitive HPC clusters.

undergoing EHT $\left(\mathrm{CD} 31^{+} \mathrm{CD} 43^{+} \mathrm{CD} 44^{+}\right)$or became floating after EHT $\left(\mathrm{CD} 43^{+} \mathrm{CD} 44^{+}\right)$significantly increased in condition with ROCK inhibition (ROCKi) (Fig. 7h). Notably, the total number of floating definitive progenitors was also significantly higher in the presence of ROCK inhibitors (right lower panel, Fig. 7h). Functionally, $\mathrm{CD} 43^{+}$HSPCs generated under ROCKi treatment produced more human $\mathrm{CD} 45^{+}$cells containing both myeloid and lymphoid cells upon co-culturing with MS5 (Fig. 7i). In all, we reveal that ROCK inhibition greatly promotes human definitive hematopoiesis to generate multipotent progenitors in hPSC differentiation.

\section{Discussion}

Given the therapeutic potential of blood and the immune cells such as NK and T cells, the generation of definitive multi-potent HSPCs in vitro, particularly from human PSCs has been a long-sought goal. However, HPCs derived from hPSCs have very limited potential in generating multiple blood lineages, particularly a very limited lymphoid potential ${ }^{23,24,27,28}$. During early hematopoietic development, the lymphoid lineages are mainly generated through definitive hematopoiesis ${ }^{14-16}$. Limited knowledge on the characterization of human early definitive hematopoiesis largely impedes the progress to generate definitive HSPCs in vitro. Here, we resolved cell populations and characterized the human definitive and primitive hematopoiesis at the earliest hematopoiesis both in hESC differentiation and early embryonic development. We identified CD44 to discriminate two distinct hematopoiesis at the earliest stage of human hematopoietic development, which allows us to generate definitive HSPCs through hPSC differentiation. Functionally, the generated definitive HSPCs from hESCs hold the multi-potency to give rise to various blood cells such as $\mathrm{Er} / \mathrm{Mk}$, My, and more importantly the immune cells such as NK, T cells, as validated by single-cell assays. Strikingly, these hESCsderived definitive HSPCs exhibit homing and migration and contribute multiple lineages in vivo upon transplantation, including My, Mk-Er, and the lymphoid lineages such as NK and B cells, thus supporting their therapeutic potential.
Notably, the definitive HSPCs derived from hESCs largely resemble human early embryonic HSPCs in terms of intrinsic lineage potential, with $\mathrm{Er} / \mathrm{My} / \mathrm{Mk}$ predominance and lower Ly representation (Fig. 4) ${ }^{33}$. This consistency supports our findings as a valuable model to investigate human early hematopoiesis. Indeed, the earliest primitive and definitive hematopoiesis at EHT displays highly similar phenotype in hESC differentiation and human embryo (Figs. 5, 6). Furthermore, we characterized dynamic gene expressions in human definitive and primitive hematopoiesis and identified signaling pathways that differentially regulate definitive and primitive hematopoiesis $^{35-38}$ (Fig. 7). Among these pathways, ROCK signaling has not been known to regulate human hematopoietic development. Strikingly, inhibition of ROCK greatly enhances human definitive hematopoiesis in hESC differentiation (Fig. 7). We thus established an enhanced approach to generate and then isolate multipotent definitive HSPCs from human PSCs. We lastly confirmed that CD44-labeled definitive HSPCs could also be generated from human urine cell-derived iPSCs (Supplementary Fig. S2). Thus, our studies not only provide a valuable model to understand human hematopoietic development and also a firm basis to generate patient's specific blood and immune cells for clinical purposes.

\section{Materials and methods \\ hPSCs culture and maintenance}

All human PSC cell lines were maintained on Matrigel (1:100 dilution; BD)-coated plates in mTeSR1 medium (Stem Cell Technologies) supplemented with $1 \%$ penicillinstreptomycin (Hyclone). Medium was changed every day and cells were passaged 1:3 onto fresh Matrigel-coated plates every 3 days using $0.5 \mathrm{mM}$ ethylenediaminetetraacetic acid disodium salt (EDTA-2Na). All of the human PSC cell lines mentioned above were maintained at $37^{\circ} \mathrm{C}$, $20 \% \mathrm{O}_{2}$ and $5 \% \mathrm{CO}_{2}$ condition and had been tested to be free of mycoplasma contamination.

\section{Hematopoietic differentiation of hPSCs}

Prior to differentiation, the hPSCs should be $80 \% \sim 90 \%$ confluent and were dissociated into single cells using 
a

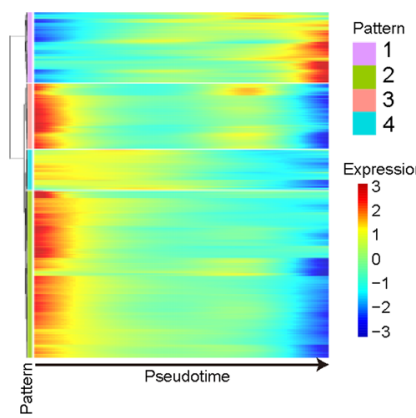

b

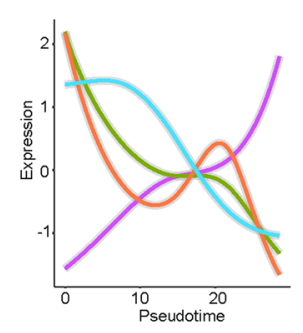

c

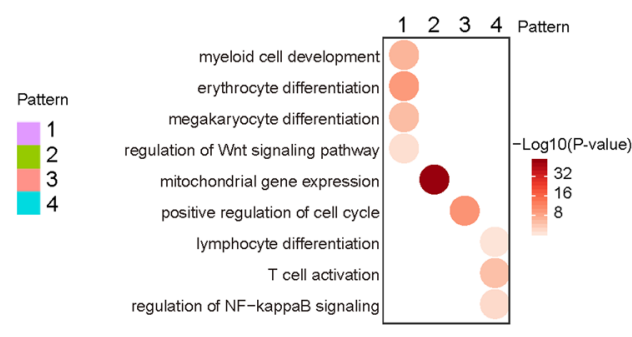

d

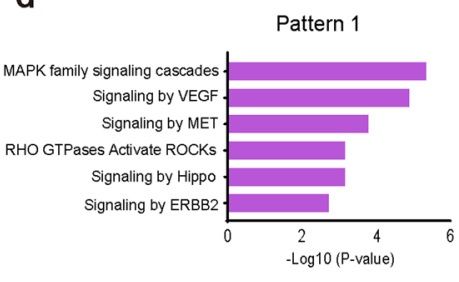

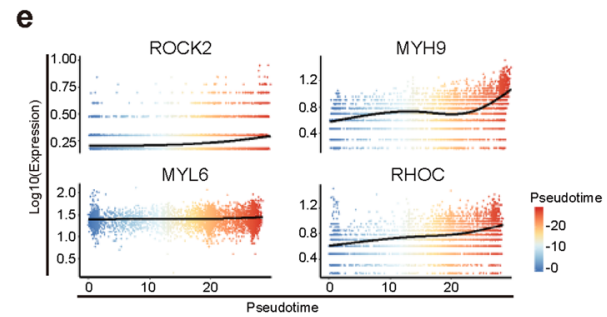

f

g

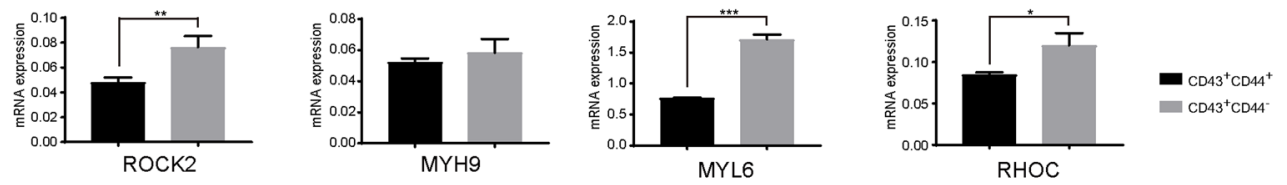

h
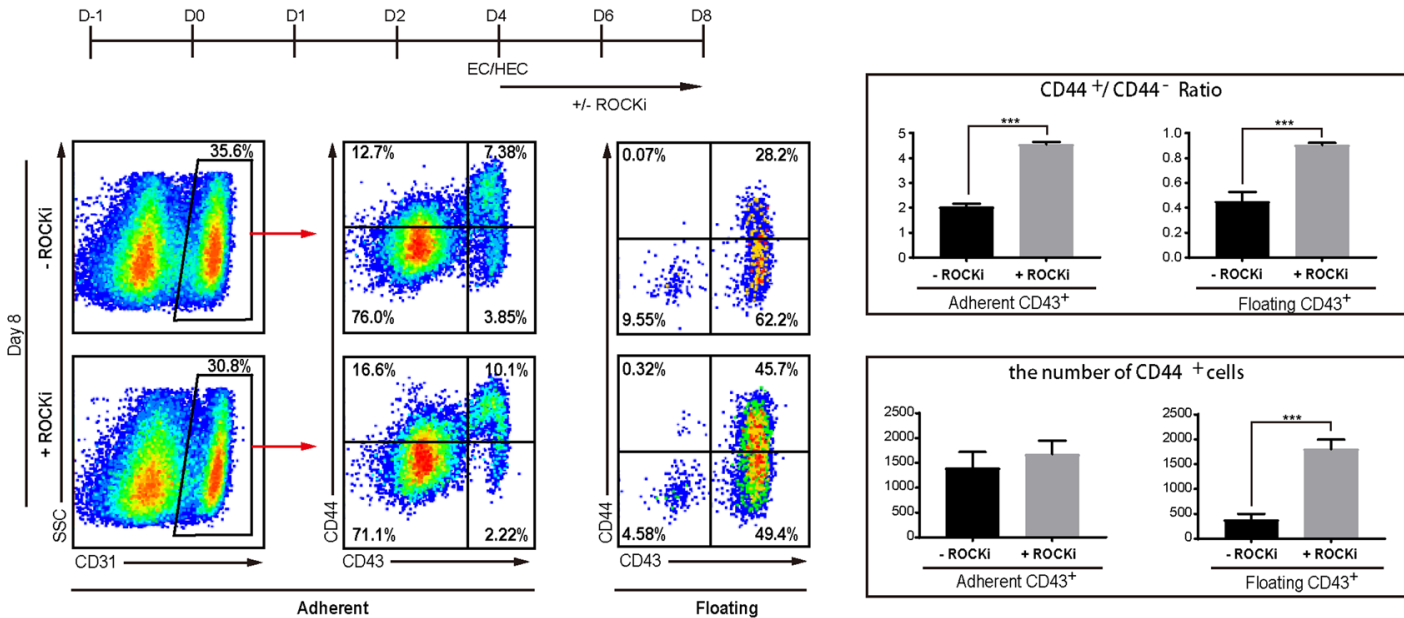

i
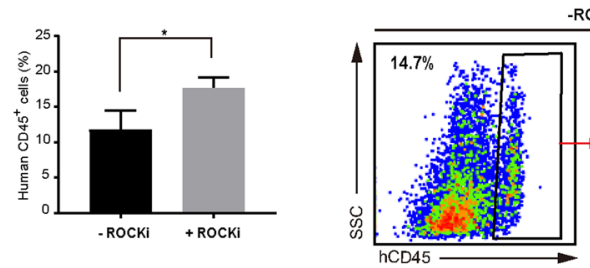

$-\mathrm{ROCK}$

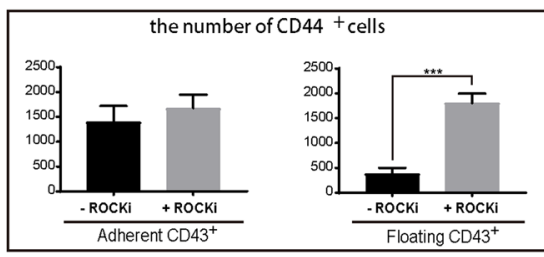

Floating $\mathrm{CD} 3^{+}$cells co-culture with MS5

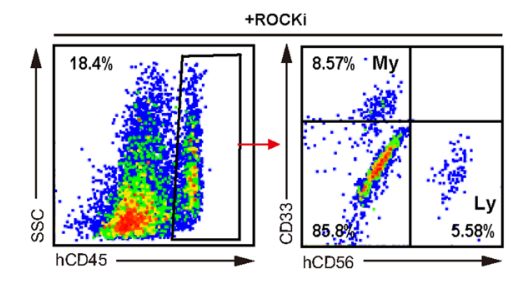

Fig. 7 (See legend on next page.) 


\begin{abstract}
(see figure on previous page)
Fig. 7 ROCK inhibition promotes the generation of definitive HSPCs from hESCs. $\mathbf{a}$, $\mathbf{b}$ Expression profiles of genes significantly upregulated or downregulated along the pseudotime progression. Based on changing profile, genes were clustered hierarchically into four different patterns (b). Lines show the mean values of scaled expression levels (TPM, z-normalized) in each pattern. c Featured GO terms enriched in genes of each pattern. Color displays P-value (Log-scaled) of GO terms. d KEGG enriched genes in pattern 1 indicated in $\mathbf{b}$. e Expression profiles of ROCK pathway-related genes along the pseudotime. Each dot represents one cell and color represents pseudotime value. Line shows the mean expression level (TPM, Logscaled). $\mathbf{f}$ Violin plots show expression levels (TPM, Log-scaled) of these ROCK signaling genes in early definitive and primitive HSPCs identified in human embryo. $\mathbf{g}$ qRT-PCR analysis of the ROCK pathway-related genes in $\mathrm{CD} 43^{+} \mathrm{CD} 44^{+}$or $\mathrm{CD} 43^{+} \mathrm{CD} 44^{-} \mathrm{HPCs}$ sorted at differentiation day 8 . The significance level was determined using unpaired two-tailed Student's $t$-tests, ${ }^{*} P<0.05,{ }^{* *} P<0.01,{ }^{* * *} P<0.001$. The data represent mean \pm SD from three independent replicates $(n=3)$. $\mathbf{h}$ Analysis of ROCK inhibition in hESC differentiation. The chemical inhibitor, Y-27632, was added in EHT medium at differentiation day 4 as indicated. The adherent or floating cells at differentiation day 8 were analyzed by FACS for the expression of CD31, CD43, and CD44. Right panel: quantitative data of each indicated population at differentiation day 8 of hESCs. The significance level was determined using unpaired two-tailed Student's $t$-tests, ${ }^{* *} P<0.001$. The data represent the mean \pm SD from three independent replicates $(n=3)$. i Function Analysis of $\mathrm{CD}_{4} 3^{+}$HSPCs generated under ROCKi treatment. Y-27632 was added in EHT medium at differentiation day 4 as indicated. The floating CD43 ${ }^{+}$cells at differentiation day 8 were counted and co-cultured with MS5 with 10,000 cells per well. Human CD45 ${ }^{+}$cells were gated 2 weeks after differentiation and analyzed for the known lineage markers as indicated. My, myeloid; Ly, lymphoid. The significance level was determined using unpaired two-tailed Student's $t$-tests, ${ }^{*} P<0.05$. The data represent mean \pm SD from three independent replicates $(n=3)$.
\end{abstract}

Accutase (Sigma). And then cells were plated onto Growth Factor Reduced (GFR) Matrigel (1:100 dilution; BD)-coated six-well plates at a proper initial density of about $3 \times 10^{5} /$ well. Especially, in order to inhibit hPSCs apoptosis, thiazovivin $(0.1 \mu \mathrm{M}$, Selleck) was added in the medium. After 24-36 h culture, the cells were about $10 \%$ confluent and this day was designated as day 0 (D0). Then, the hPSCs were induced for stepwise differentiation in basal medium (BM) supplemented with cytokines and inhibitors in the following days. D0-D1: $40 \mathrm{ng} / \mathrm{ml} \mathrm{BMP4}$ (Peprotech), $30 \mathrm{ng} / \mathrm{ml}$ ACTIVIN A (Sino Biological), $20 \mathrm{ng} / \mathrm{ml}$ bFGF (Sino Biological), $6 \mu \mathrm{M}$ CHIR99021 (Selleck) and $10 \mu$ M LY294002 (Selleck); D1-D2: $30 \mathrm{ng} / \mathrm{ml}$ BMP4, $1 \mu \mathrm{M}$ A8301 (Selleck) and $2 \mu \mathrm{M}$ IWR-1-endo (Selleck); D2-D4: $40 \mathrm{ng} / \mathrm{ml}$ VEGF (Sino Biological) and $50 \mathrm{ng} / \mathrm{ml}$ bFGF; D4-D8: 40 ng/ml VEGF, $50 \mathrm{ng} / \mathrm{ml}$ bFGF, $10 \mu \mathrm{M}$ SB431542 (Selleck), $10 \mathrm{ng} / \mathrm{ml}$ SCF (Peprotech), $50 \mathrm{ng} / \mathrm{ml}$ TPO (Sino Biological), $10 \mathrm{ng} / \mathrm{ml}$ IL3 (Sino Biological), $50 \mathrm{ng} / \mathrm{ml}$ IL6 (Sino Biological) and $50 \mathrm{ng} / \mathrm{ml}$ FLT3L (Peprotech). BM: DMEM/F12 (GIBCO) + 1\% penicillin-streptomycin (Hyclone) $+1 \%$ insulintransferrin-selenium (ITS, GIBCO) $+70 \mu \mathrm{g} / \mathrm{ml}$ vitamin $\mathrm{C}$ (Vc, 2-Phospho-L-ascorbic acid trisodium salt solution, Sigma). Particularly, the osmotic pressure of the HDM was adjusted by $9 \% \mathrm{NaCl}$ to about 340 . The hematopoietic differentiation medium in each step should be changed every day and the differentiating cells were differentiated in $37^{\circ} \mathrm{C}, 20 \% \mathrm{O}_{2}$ and $5 \% \mathrm{CO}_{2}$ condition.

\section{Quantitative real-time PCR (qRT-PCR)}

The total RNA was extracted from cells using the RaPure Total RNA Micro Kit (Magen) and $2 \mu$ g RNA was reversely transcribed into cDNA with a HiScript II 1st Strand cDNA Synthesis Kit (Vazyme). Then, qRT-PCR was performed with ChamQ SYBR qPCR Master Mix (Vazyme) and a CFX96 machine (Bio-Rad). GAPDH was used for normalization. All data were analyzed with 3 replicates and all primers used in this study were listed in Supplementary Table S1.

\section{CFU assay and cell morphology}

The CFU assay was performed according to the manufacturer's instruction of Methocult H4435 (Stem Cell Technologies). Firstly, an indicated number of single cells were suspended into $120 \mu \mathrm{l}$ IMDM medium supplemented with $2 \%$ FBS (Biological Industries), and then cell suspension was added into $1 \mathrm{ml}$ Methocult H4435. Next, we transferred the mixture to $35 \mathrm{~mm}$ ultra-low attachment plates (Stem Cell Technologies) and rotated the plates gently to spread methylcellulose medium over the surface of the dish. We placed three dishes within a $100 \mathrm{~mm}$ petri dish containing $3 \mathrm{~mL}$ sterile water and incubated the dishes in $37^{\circ} \mathrm{C}, 20 \% \mathrm{O}_{2}$ and $5 \% \mathrm{CO}_{2}$ condition. The CFUs were classified and calculated according to the morphology after 2 weeks. All data were analyzed with three replicates. Then, we harvested the proper number of cells from different colony types in CFU assay, rinsed them twice with DPBS (gibco) and resuspended the cells in $200 \mu \mathrm{l}$ DPBS. The morphology of the cell was showed by microscopy after cytospin preparation and staining with May-Grunwald-Giemsa.

\section{Flow cytometry and cell sorting}

A single-cell suspension was prepared by Accutase (Sigma) and filtered through $70 \mu \mathrm{m}$ filter. Then, cells were stained by multicolor antibody combinations in DPBS supplemented with $2 \% \mathrm{FBS}$ and incubated at $4{ }^{\circ} \mathrm{C}$ for 20-30 min. The cells were sorted by the Aria II (BD) or Moflo (Beckman). Antibodies were listed in Supplementary Table S2.

\section{In vitro lympho-myeloid lineage differentiation at bulk}

MS-5 cells were seeded onto $0.1 \%$ gelatin-coated 24well plate at an initiating density of $2 \times 10^{4} /$ well in $\alpha$ - 
MEM medium (Thermo Fisher) supplemented with 10\% FBS (Gibco), 1\% penicillin-streptomycin (Hyclone) and $1 \%$ GlutaMAX $^{\mathrm{TM}}$ (Gibco). Twenty-four hours after plating of MS-5 stroma, $1 \times 10^{4}$ cells were added into each well in the presence of $0.1 \mu \mathrm{M}$ DuP-697 (Biovision), $20 \mathrm{ng} / \mathrm{ml} \mathrm{SCF}$ (Peprotech), $10 \mathrm{ng} / \mathrm{ml} \mathrm{G-CSF}$ (Peprotech), $10 \mathrm{ng} / \mathrm{ml}$ FLT3L (Peprotech), $10 \mathrm{ng} / \mathrm{ml} \mathrm{IL-2} \mathrm{(Peprotech),}$ and $10 \mathrm{ng} / \mathrm{ml} \mathrm{IL-15}$ (Sino Biological). Half of the medium was changed twice every week and cocultures were transferred onto fresh MS-5 stroma every two weeks through $40 \mu \mathrm{m}$ filter to remove the stromal cells. All of the cells in each well were harvested and analyzed by flow cytometry at week 1 and week 4 . The antibodies used to read the lineage outputs in this assay were anti-human CD45-PE, anti-human CD14-PE-Cy7, anti-human CD15APC, anti-human CD19-FITC, anti-human CD56-PerCPCy5.5, anti-human CD45-APC, anti-human CD235aFITC, and anti-human CD41-PE.

\section{In vitro lympho-myeloid lineage differentiation at single- cell level}

For single-cell analysis, MS-5 cells were seeded onto $0.1 \%$ gelatin-coated 96 -well plate at an initiating density of $2.5 \times 10^{3} /$ well in $\alpha$-MEM medium (Thermo Fisher) supplemented with 10\% FBS (Gibco), 1\% penicillinstreptomycin (Hyclone), 1\% GlutaMAX ${ }^{\mathrm{TM}}$ (Gibco), $0.1 \mu \mathrm{M}$ DuP-697 (Biovision), $20 \mathrm{ng} / \mathrm{ml} \mathrm{SCF}$ (Peprotech), $10 \mathrm{ng} / \mathrm{ml} \mathrm{G-CSF}$ (Peprotech), $10 \mathrm{ng} / \mathrm{ml}$ FLT3L (Peprotech), $10 \mathrm{ng} / \mathrm{ml} \mathrm{IL-2} \mathrm{(Peprotech)} \mathrm{and} 10 \mathrm{ng} / \mathrm{ml} \mathrm{IL-15}$ (Sino Biological). Twenty-four hours later, single CD44-positive hESC-HPCs were sorted into 96-well plates and half of the medium was changed every week. After culturing for 3-4 weeks, all of the cells in each well were resuspended by physical dissociation and analyzed by flow cytometry, and wells with more than 15 human $\mathrm{CD} 235 \mathrm{a}^{+}, \mathrm{CD} 41^{+}$, $\mathrm{CD}_{3} 3^{+} / \mathrm{CD}_{11 \mathrm{~b}^{+}}$, or $\mathrm{CD} 56^{+}$cells were considered positive. The antibodies used to read the lineage outputs in this assay were anti-human CD45-PE-Cy7, anti-human CD33-PE, anti-human CD56-APC.

\section{In vitro $\mathrm{T}$ lineage differentiation}

OP9-hDL4 cells were seeded onto a 24-well plate coated by $0.1 \%$ gelatin at a density of $2 \times 10^{4} /$ well in $\alpha$-MEM medium (Thermo Fisher) supplemented with 20\% FBS (Gibco), 1\% penicillin-streptomycin (Hyclone) and 1\% GlutaMAX $^{\mathrm{TM}}$ (Gibco). One day after plating of OP9hDL4 cells, $1 \times 10^{4}$ cells were deposited into each well containing $10 \mathrm{ng} / \mathrm{ml} \mathrm{SCF}$ (Peprotech), $5 \mathrm{ng} / \mathrm{ml}$ FLT3L (Peprotech) and $5 \mathrm{ng} / \mathrm{ml} \mathrm{IL-7} \mathrm{(Sino} \mathrm{Biological).} \mathrm{Half} \mathrm{of} \mathrm{the}$ medium was changed twice every week. Harvested cells in each well were analyzed by flow cytometry at week 2 and the antibodies used to read the lineage outputs in this assay were as follows: anti-human CD45-PE, anti-human CD4-PE-Cy7, anti-human CD8-APC-Cy7.

\section{Mice and in vivo myeloid lineage differentiation}

NSI (NOD/SCID IL2 $\mathrm{rg}^{-1-}$ ) mice were purchased from Peng Li's lab in Guangzhou Institutes of Biomedicine and Health, Chinese Academy of Sciences (GIBH, CAS). Mice were bred and housed in the SPF-grade animal care facility of the GIBH. All the animal experiments had been approved by the Animal Ethnical Committee of GIBH and performed according to the ISSCR guidelines. Mice (6- to 8-week old) were sublethally irradiated (1.2-1.5 Gry) 12$24 \mathrm{~h}$ before transplantation. Then, mice were anesthetized by intraperitoneal injection with 2,2,2-Tribromoethanol (Sigma) and cells were transplanted intratibially into the mice. In brief, an insulin needle was used to drill the tibia firstly, and then a volume of $20-25 \mu \mathrm{l}$ cells were transplanted into the tibia with another insulin needle. After two weeks, peripheral blood was isolated from the orbital venous plexus, and then mice were euthanized and the $\mathrm{BM}$ in tibia was flushed by PBS with a $1 \mathrm{ml}$ needle. The potential contamination of mouse CD45+ cells had been excluded through strict gating using negative control. For lineage differentiation analysis, the peripheral blood and marrow were processed by ACK lysis buffer and then stained using the following antibodies: anti-human CD45$\mathrm{PE}$, anti-human CD45-PE-Cy7, anti-human CD14-PECy7, anti-human CD15-APC, anti-human CD11b-APC, anti-human CD19-FITC, anti-human CD56-APC-Cy7, anti-human CD235a-PE, anti-human CD235a-FITC, antihuman CD41-PE-Cy5, and anti-human CD41-PE.

\section{LDA assay}

For the limiting-dilution assay (LDA), different doses of CD44 positive hESC-HPCs $(5,10,20$, and 50 cells) were sorted into 96-well plates pre-plated with $2.5 \times 10^{3}$ MS-5 cells per well supplemented with 10\% FBS (Gibco), 1\% penicillin-streptomycin (Hyclone), 1\% GlutaMAX ${ }^{\mathrm{TM}}$ (Gibco), $0.1 \mu \mathrm{M}$ DuP-697 (Biovision), $20 \mathrm{ng} / \mathrm{ml}$ SCF (Peprotech), $10 \mathrm{ng} / \mathrm{ml}$ G-CSF (Peprotech), $10 \mathrm{ng} / \mathrm{ml}$ FLT3L (Peprotech), $10 \mathrm{ng} / \mathrm{ml}$ IL-2 (Peprotech) and $10 \mathrm{ng} /$ ml IL-15 (Sino Biological). Half of the medium was changed every week. After culturing for 3-4 weeks, all of the cells in each well were resuspended by physical dissociation and analyzed by flow cytometry. The antibodies used to read the lineage outputs in this assay were antihuman CD45-PE-Cy7, anti-human CD33-PE, antihuman CD56-APC. Frequency calculations were performed using ELDA software (http://bioinf.wehi.edu.au/ software/elda/).

\section{Immunofluorescence}

The adherent cells were fixed by $4 \%$ paraformaldehyde (PFA) for $30 \mathrm{~min}$ and incubated with primary antibodies overnight at $4{ }^{\circ} \mathrm{C}$. After that, cells were washed with PBS and incubated with specific secondary antibodies. Nuclei were stained with DAPI. Stained cells were observed using 
a LSM800 confocal microscope (Carl Zeiss). Antibodies were listed in Supplementary Table S2.

\section{Single-cell RNA sequencing}

Fresh mobilized PB samples were obtained as a gift from the Department of Hematology, The Third Affiliated Hospital, Sun Yat-sen University. They were processed within $24 \mathrm{~h}$ after received. Mononuclear cells were isolated firstly by removing of red blood cells using ACK lysis buffer, and then CD34 ${ }^{+}$fraction was separated by CD34 Microbead kit and magnetic-activated cell-sorting separation columns (Miltenyi Biotec) according to the manufacturer's instruction. For cell sorting, PB CD34 ${ }^{+}$ fraction was stained with human hematopoietic lineage FITC cocktail (CD4, CD8, CD11b, CD14, CD19, CD20, CD56, CD235a, and CD10), anti-human CD34-PercpCy5.5 and anti-human CD38-APC and sorted by the Aria II (BD). Human ESCs were differentiated into blood lineages in a monolayer, defined condition, and the floating blood cells at differentiation day 8 were collected and sorted by CD43. We have conducted multiple differentiation experiments and tested the CD43 expression of hESC-HPC to ensure the repeatability and consistency of the experiment, and then sorted $\mathrm{CD} 43^{+}$cells from one experiment for single-cell RNA sequencing. The sorted cells were analyzed by $10 \times$ genomics for single-cell RNA sequencing (scRNA-seq). The single-cell libraries were prepared following the Chromium Single Cell 3' Reagent Kits User Guide, quantified by Quant-iT dsDNA Assay Kit with high sensitivity (Thermo Fisher) on Qubit 2.0, and in the end, sequenced on illumina NextSeq 500.

\section{scRNA-seq data processing}

$10 \times$ sequencing data were processed with cellranger (version 2.1.1) with default parameters. Then we performed quality control to filter low-quality cells. Cells with UMI counts range in Mean \pm 4 SD (log transformed) were kept; cells with more than $10 \%$ of reads mapped to mitochondrial genes were removed. For hESC-derived cell samples, the Mean Reads per Cell is 83238, the Median Gene numbers per Cell is 3932, the Median UMI Counts per Cell is 25354; For the PB HSPC sample, the Mean Reads per Cell is 71890, the Median Genes per Cell is 1953, the Median UMI Counts per Cell is 7718 .

\section{Dimension reduction and clustering}

We used Seurat (version 3.0.0) to normalize data and reduce dimension. Only genes expressed in at least three cells were retained and only cells that express more than 200 genes were retained. Then, FindVariableGenes function was applied to select highly variable genes on logtransformed data. SCTransform function was used to normalize data. PCA was performed using highly variable genes. JackStraw function was used to select significant
PCs, and PCs with $P$-value $<0.05$ were used for downstream analyses. Cells were projected in two-dimension using RunTSNE function. In integrated analysis of PB-D8 sample, FindClusters function was used to perform clustering with parameter "resolution" $=0.04$, resulting in three clusters.

\section{Identification of DEGs}

DEGs were identified using FindMarkers function. Genes with log-fold-change more than $0.1, P$-value $<0.01$, and the difference of pct 1 and pct 2 more than 0.05 were selected as DEGs.

\section{Pseudotime trajectory analysis}

Pseudotime trajectory analysis was performed with Monocle (version 2.10.1). Genes with more than fitted dispersion evaluated using dispersionTable function were identified as highly variable genes. The Discriminative Dimensionality Reduction with Trees (DDRTree) method was used to reduce dimensions. Significant DEGs along pseudotime were identified by differentialGeneTest function.

\section{d-cyto Analysis}

d-cyto Analysis was inspired by flow cytometry. It takes RNA expression levels as inputs. The Axis panel was divided into tiles and the color of each tile represents the number of cells in it. Cells were gated depending on geneexpression levels. Percentages of cells in gate were calculated.

\section{GO analysis}

GO enrichment was performed using clusterProfiler (version 3.10.1).

\section{Statistical analysis}

Data were presented as mean \pm SD, and statistics were determined by unpaired two-tailed Student's $t$-test ( $t$ test). $P$ value $<0.05$ was considered statistically significant. " $P<0.05 ;{ }^{* *} P<0.01 ; * * * P<0.001$. No statistical method was used to pre-determine the sample size. No samples were excluded for any analysis. No randomization was used for allocating animal groups. No blinding done in animal experiments.

\section{Acknowledgements}

We thank the lab members in GIBH for their kindly help. We thank Dr. Xinwen Chen for critical comments and encouragement for this work. We thank

Dr. Hui Cheng for MS5 cells used for experiments. This work was granted by the National Key Research and Development Program of China, Stem Cell and Translational Research (2017YFA0102600); Strategic Priority Research Program of Chinese Academy of Sciences (Grant No. XDA16030504); Science and Technology Planning Project of Guangdong Province, China

(2017B030314056); the Frontier and Key Technology Innovation Special Grant from the Department of Science and Technology of Guangdong Province (2016B030230002, 2016B030229008); the National Natural Science Foundation of China (31801220, 31971374); Guangzhou Science and Technology Program 
General project (20180304001, 201904020045, 201904010462); Innovative Team Program of Guangzhou Regenerative Medicine and Health Guangdong Laboratory (2018GZR110104005), the Informationization Special Project of Chinese Academy of Sciences "E-Science Application for Knowledge Discovery in Stem Cells (XXH13506-203), the Open Research Funds of the State Key Laboratory of Ophthalmology (2019KF06); the Guangdong Province Special Program for Outstanding Talents (to G.P., 2019JC05Y463).

\section{Author details}

${ }^{1}$ CAS Key Laboratory of Regenerative Biology, Joint School of Life Sciences, Guangzhou Institutes of Biomedicine and Health, Chinese Academy of Sciences, Guangzhou Medical University, ,Guangzhou Guangdong 510530, China. ${ }^{2}$ Guangdong Provincial Key Laboratory of Stem Cell and Regenerative Medicine, South China Institute for Stem Cell Biology and Regenerative Medicine, Guangzhou Institutes of Biomedicine and Health, Chinese Academy of Sciences, ,Guangzhou Guangdong 510530, China. ${ }^{3}$ University of Chinese Academy of Sciences, Beijing 100049, China. ${ }^{4}$ Department of Hematology, The Third Affiliated Hospital, Sun Yat-sen University, ,Guangzhou Guangdong 510630, China. ${ }^{5}$ State Key Laboratory of Experimental Hematology, Fifth Medical Center of Chinese PLA General Hospital, Beijing 100071, China. ${ }^{6}$ Hefei Institute of Stem Cell and Regenerative Medicine, Guangzhou Institutes of Biomedicine and Health, Chinese Academy of Sciences, ,Guangzhou Guangdong 510530, China. ${ }^{7}$ Institute for Stem Cell and Regeneration, Chinese Academy of Sciences, Beijing 100101, China

\section{Author contributions}

G.P. and Y.Z. designed the project and analyzed the data. G.P., Y.Z., T.W., and J.G. Wrote the manuscript. T.W. analyzed the single-cell RNA sequencing data. Y.Z., T.W., and J.G. designed and performed most experiments and analyzed result data. J.G. and Z.Z. performed the animal experiments. H.L. and Q.C. performed the single-cell RNA sequencing. J.T., Y.M., Y.Z., Y.L., and J.L. assisted with the FACS. J.T., D.Z., and M.Z. performed the qRT-PCR assays. Y.F. and J.Z. assisted with animal experiments. B.K., J.W., and M.G. assisted with the cellsorting assays. B.K. and T.Z. assisted with the CFU assay. K.H., Y.L., B.L., X.Z., J.C., D.P., J.N., and B.L. gave experiment suggestions or provided experiment materials for this research. All authors read and approved the final manuscript.

\section{Data availability}

The RNA-Seq data have been deposited in the Gene Expression Omnibus database under the accession code GSE148215. The authors declare that all data supporting the findings of this study are available within the article and its supplementary information files or from the corresponding author (Dr. Guangjin Pan, pan_guangjin@gibh.ac.cn) upon reasonable request.

\section{Conflict of interest}

The authors declare that they have no conflict of interest.

\section{Publisher's note}

Springer Nature remains neutral with regard to jurisdictional claims in published maps and institutional affiliations.

Supplementary Information accompanies the paper at (https://doi.org/ 10.1038/s41421-020-00213-6).

Received: 15 July 2020 Accepted: 2 September 2020

Published online: 01 December 2020

\section{References}

1. Laurenti, E. \& Gottgens, B. From haematopoietic stem cells to complex differentiation landscapes. Nature 553, 418-426 (2018).

2. Haas, S., Trumpp, A. \& Milsom, M. D. Causes and consequences of hematopoietic stem cell heterogeneity. Cell Stem Cell 22, 627-638 (2018).

3. Cvejic, A. Mechanisms of fate decision and lineage commitment during haematopoiesis. Immunol. Cell Biol. 94, 230-235 (2015).

4. Manz, M. G., Miyamoto, T., Akashi, K. \& Weissman, I. L. Prospective isolation of human clonogenic common myeloid progenitors. Proc. Natl Acad. Sci. USA 99, 11872-11877 (2002)
5. Galy, A., Travis, M., Cen, D. \& Chen, B. Human T, B, natural killer, and dendritic cells arise from a common bone marrow progenitor cell subset. Immunity $\mathbf{3}$ 459-473 (1995).

6. Doulatov, S. et al. Revised map of the human progenitor hierarchy shows the origin of macrophages and dendritic cells in early lymphoid development. Nat. Immunol. 11, 585-U552 (2010).

7. Laurenti, E. et al. The transcriptional architecture of early human hematopoiesis identifies multilevel control of lymphoid commitment. Nat. Immunol. 14, 756-763 (2013)

8. Notta, F., Zandi, S., Takayama, N., Dobson, S. \& Dick, J. E. Distinct routes of lineage development reshape the human blood hierarchy across ontogeny. Science 351, aab2116 (2015)

9. Velten, L. et al. Human haematopoietic stem cell lineage commitment is a continuous process. Nat. Cell Biol. 19, 271-281 (2017).

10. Grun, D. et al. De novo prediction of stem cell identity using single-cell transcriptome data. Cell Stem Cell 19, 266-277 (2016).

11. Paul, F. et al. Transcriptional heterogeneity and lineage commitment in myeloid progenitors. Cell 163, 1663-1677 (2015).

12. Majeti, R., Park, C. Y. \& Weissman, I. L. Identification of a hierarchy of multipotent hematopoietic progenitors in human cord blood. Cell Stem Cell 1, 635-645 (2007).

13. Karamitros, D. et al. Single-cell analysis reveals the continuum of human lympho-myeloid progenitor cells. Nat. Immunol. 19, 85-97 (2018).

14. Medvinsky, A. \& Dzierzak, E. Definitive hematopoiesis is autonomously initiated by the AGM region. Cell 86, 897-906 (1996).

15. Muller, A. M., Medvinsky, A., Strouboulis, J., Grosveld, F. \& Dzierzak, E. Development of hematopoietic stem-cell activity in the mouse embryo. Immunity $\mathbf{1}$, 291-301 (1994).

16. Bertrand, J. Y. et al. Haematopoietic stem cells derive directly from aortic endothelium during development. Nature 464, 108-111 (2010).

17. Boisset, J. C. et al. In vivo imaging of haematopoietic cells emerging from the mouse aortic endothelium. Nature 464, 116-120 (2010).

18. Eilken, H. M., Nishikawa, S. \& Schroeder, T. Continuous single-cell imaging of blood generation from haemogenic endothelium. Nature 457, 896-900 (2009).

19. Jagannathan-Bogdan, M. \& Zon, L. I. Hematopoiesis. Development $\mathbf{1 4 0}$ 2463-2467 (2013).

20. Sturgeon, C. M., Ditadi, A., Awong, G., Kennedy, M. \& Keller, G. Wnt signaling controls the specification of definitive and primitive hematopoiesis from human pluripotent stem cells. Nat. Biotechnol. 32, 554-561 (2014).

21. Garcia-Alegria, E. et al. Early human hemogenic endothelium generates primitive and definitive hematopoiesis in vitro. Stem Cell Rep. 11, 1061-1074 (2018).

22. Ditadi, A. et al. Human definitive haemogenic endothelium and arterial vascular endothelium represent distinct lineages. Nat. Cell Biol. 17, 580-591 (2015).

23. Uenishi, G. I. et al. NOTCH signaling specifies arterial-type definitive hemogenic endothelium from human pluripotent stem cells. Nat. Commun. 9, 1828 (2018).

24. Vodyanik, M. A., Thomson, J. A. \& Slukvin, I. I. Leukosialin (CD43) defines hematopoietic progenitors in human embryonic stem cell differentiation cultures. Blood 108, 2095-2105 (2006).

25. Dou, D. R. et al. Medial HOXA genes demarcate haematopoietic stem cell fate during human development. Nat. Cell Biol. 18, 595-606 (2016).

26. Daniel, M. G., Pereira, C. F., Lemischka, I. R. \& Moore, K. A. Making a hematopoietic stem cell. Trends Cell Biol. 26, 202-214 (2016).

27. Huang, $K$. et al. Generation and analysis of GATA2w/eGFP human ESCs reveal ITGB3/CD61 as a reliable marker for defining hemogenic endothelial cells during hematopoiesis. Stem Cell Rep. 7, 854-868 (2016).

28. Zhang, T. et al. Vitamin C-dependent lysine demethylase 6 (KDM6)-mediated demethylation promotes a chromatin state that supports the endothelial-tohematopoietic transition. J. Biol. Chem. 294, 13657-13670 (2019).

29. Loh, K. M. et al. Mapping the pairwise choices leading from pluripotency to human bone, heart, and other mesoderm cell types. Cell 166, 451-467 (2016).

30. Kennedy, M. et al. T lymphocyte potential marks the emergence of definitive hematopoietic progenitors in human pluripotent stem cell differentiation cultures. Cell Rep. 2, 1722-1735 (2012).

31. Pan, $\mathbf{X}$. et al. Graded levels of GATA-1 expression modulate survival, proliferation, and differentiation of erythroid progenitors. J. Biol. Chem. 280 22385-22394 (2005). 
32. Armstrong, J. A. \& Emerson, B. M. NF-E2 disrupts chromatin structure at human beta-globin locus control region hypersensitive site 2 in vitro. Mol. Cell Biol. 16, 5634-5644 (1996).

33. Popescu, D. M. et al. Decoding human fetal liver haematopoiesis. Nature $\mathbf{5 7 4}$, 365-371 (2019).

34. Zeng, Y. et al. Tracing the first hematopoietic stem cell generation in human embryo by single-cell RNA sequencing. Cell Res. 29, 881-894 (2019).

35. Geest, C. R. \& Coffer, P. J. MAPK signaling pathways in the regulation of hematopoiesis. J. Leukocyte Biol. 86, 237-250 (2009).
36. Saadatzadeh, M. R., Bijangi-Vishehsaraei, K., Kapur, R. \& Haneline, L. S. Distinct roles of stress-activated protein kinases in Fanconi anemia-type C-deficient hematopoiesis. Blood 113, 2655-2660 (2009).

37. Khadilkar, R. J. \& Tanentzapf, G. Septate junction components control Drosophila hematopoiesis through the Hippo pathway. Development 146, https:// doi.org/10.1242/dev.166819 (2019).

38. Martin, R. et al. SCL interacts with VEGF to suppress apoptosis at the onset of hematopoiesis. Development 131, 693-702 (2004). 